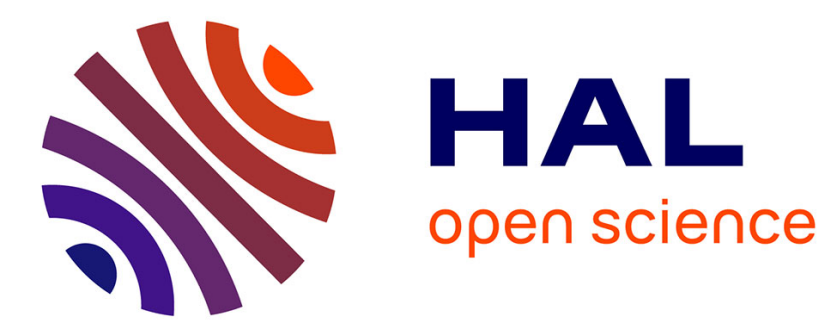

\title{
La grange de la léproserie Saint-Lazare de Beauvais
}

Frédéric Epaud

\section{To cite this version:}

Frédéric Epaud. La grange de la léproserie Saint-Lazare de Beauvais. Archéologie médiévale, 2014, 44, pp.51-70. halshs-01243347

\section{HAL Id: halshs-01243347 https://shs.hal.science/halshs-01243347}

Submitted on 29 May 2018

HAL is a multi-disciplinary open access archive for the deposit and dissemination of scientific research documents, whether they are published or not. The documents may come from teaching and research institutions in France or abroad, or from public or private research centers.
L'archive ouverte pluridisciplinaire HAL, est destinée au dépôt et à la diffusion de documents scientifiques de niveau recherche, publiés ou non, émanant des établissements d'enseignement et de recherche français ou étrangers, des laboratoires publics ou privés. 


\title{
La grange de la léproserie Saint-Lazare de Beauvais
}

\author{
Frédéric ÉPAUD*
}

Mots-clés : léproserie, grange, charpente, bois, marque, dendrochronologie

Résumé : Implantée aux abords de la ville de Beauvais, la léproserie Saint-Lazare comprenait, dans un vaste enclos compartimenté en trois espaces fonctionnels (administratif/lépreux/agricole), de nombreux bâtiments du XII et XIIIe siècle. Parmi ceux-ci, une grange de près de $50 \mathrm{~m}$ de long sur $22 \mathrm{~m}$ de large, possédant une charpente intégralement conservée, a fait l'objet d'une étude archéologique pour en comprendre les techniques de mises en œuvre. Elle a démontré que, pour les besoins de la charpente, près de 760 chênes ont été abattus en automne-hiver 1219-1220. La charpente à chevrons-formant-fermes, qui repose sur deux files de grandes arcades maçonnées, définit un vaisseau central et deux collatéraux, dont l'un servait de passage charretier pour l'engrangement. Son relevé a révélé l'utilisation de principes structurels propres aux charpentes des grandes églises gothiques franciliennes et aussi l'emploi d'assemblages d'une complexité exceptionnelle, ce qui fait de cette grange un véritable laboratoire de conception et d'expérimentation des nouvelles techniques de charpenterie pour sa période.

Keywords: Leper-House, Barn, Wood-Frame, Wood, Mark, Dendrochronology

Abstract: The barn of the Saint Lazarus leper-house in Beauvais. The Saint Lazarus leper-house, established within a vast enclosure on the outskirts of Beauvais, was comprised of three functional areas (administration, lepers' residence, farm) which included numerous $12^{\text {th }}$ and $13^{\text {th }}$ century buildings. Amongst these is a barn almost $50 \mathrm{~m}$ in length and $22 \mathrm{~m}$ wide whose totally-preserved roof timbers have been examined archaeologically to understand construction techniques. Analysis shows that almost 760 oaks were felled in the autumn-winter of 1219-1220 to provide the frame-work. The roof-frame, composed of rafters forming trusses which stand upon two lines of large masonry arches, defines a central nave and two collaterals, one of which provided cart-access for storing. Study of the barn reveals the use of structural principals peculiar to the roof frames of the great gothic churches in the Paris area as well exceptionally complex assemblages, thus making it a testing-ground for conceiving and trying-out the new wood-framing techniques of the period.

Schlüsselwörter: Leprosenhaus, Scheune, Dachwerk, Holz, Abbundzeichen, Dendrochronologie

Zusammenfassung: Das Aussätzigenhaus zum hl. Lazarus, in der Nähe der Stadt Beauvais, bestand aus einer weitläufigen Einfriedigung, die in drei Teile mit unterschiedlichen Funktionen zerfiel: Verwaltung, Aufenthalt der Leprosen und Landwirtschaft. Es enthielt viele Bauten des 12. und 13. Jhs., darunter eine fast $50 \times 22 \mathrm{~m}$ große Scheune, deren Dachwerk vollständig erhalten ist. Diese wurde bauforscherisch untersucht, um ihre Bautechnik zu klären. Es zeigte sich, dass für ihr Dachwerk im Herbst und im Winter 1219/20 fast 760 Eichen gefällt wurden. Das Kehlbalkendach stützt sich auf zwei gemauerte Arkadenreihen, die ein Haupt- und zwei Nebenschiffe abgrenzen; eins von ihnen diente als Wagendurchfahrt. Die Bauaufnahme dieses Dachwerks zeigte, dass es nicht nur dieselben Bauprinzipien aufwies, wie die großen gotischen Kirchen der Ile-de-France, sondern auch Holzverbindungen von außergewöhnlicher Komplexität; damit ist diese Scheune für ihre Zeit ein wahres Experimentierfeld innovativer Dachkonstruktion.

\footnotetext{
* Chargé de recherche au CNRS, UMR 7324 CITERES, LAT, Tours.
} 
Cette étude présente la synthèse d'un travail commandé et financé en 2006 par le service archéologique municipal de Beauvais, dans le cadre du suivi des travaux d'aménagements pour la valorisation de la maladrerie ${ }^{1}$. Son objectif était de relever et de comprendre l'ensemble des techniques et des structures de charpenterie au préalable d'une restauration de la grange, en vue de son ouverture au public. Elle participe à l'encadrement et au contrôle de l'ensemble des travaux d'entretien et de restauration du site de la léproserie par le service archéologique de la ville qui en a la charge scientifique.

La fondation de la léproserie Saint-Lazare de Beauvais se rattache au vaste mouvement de création d'établissements charitables apparu en France à la fin du XI ${ }^{\mathrm{e}}$ siècle et qui s'amplifie au $\mathrm{XII}^{\mathrm{e}}$ siècle devant l'expansion de la lèpre. Sa première mention concerne la donation d'une maison à l'établissement en 1131, confirmant une création antérieure à cette date ${ }^{2}$. Comme cela était généralement accordé à la fondation d'un établissement pour recueillir rapidement des revenus en espèces et en nature, une foire lui était rattachée et se situait sur ses abords, d'après sa première mention en $1176^{3}$. Le fondateur de cette léproserie était vraisemblablement le chapitre cathédral de Beauvais ou bien l'évêque en personne puisque le maître régisseur de SaintLazare, d'après une charte de 1234, était également chanoine et nommé par le chapitre de la cathédrale Saint-Pierre ${ }^{4}$.

Le cartulaire de la léproserie mentionne pour le $\mathrm{XII}^{\mathrm{e}}$ et le $\mathrm{XIII}^{\mathrm{e}}$ siècle l'attribution de droits et de privilèges, mais surtout de nombreux dons de terres et de revenus qui ont permis à la léproserie de constituer un important domaine foncier. Outre les possessions exploitées directement par la léproserie, les terres furent regroupées par des échanges et des rachats ${ }^{5}$ autour de trois centres d'exploitation agricole ou "granges" sur Noyers, Villers-Saint-Barthélémy et Bus-Robert. Exploitées essentiellement en faire-valoir direct, ces granges tiraient leurs bénéfices de cultures céréalières (avoine et blé), de bois, de prés, de vignes et aussi de l'élevage de chevaux, de porcs, de brebis et de vaches. La léproserie Saint-Lazare possédait à Beauvais au moins 13 maisons et percevait également le cens sur plus de 60 maisons au XIII ${ }^{\mathrm{e}}$ siècle. L'importance des revenus tirés de ce domaine, des droits et des possessions immobilières, tout comme de l'exercice de la basse et moyenne justice confèrent à cet établissement pour le XIII ${ }^{\mathrm{e}}$ siècle un statut à part, plus proche de celui d'une abbaye que de celui d'une léproserie, généralement moins richement dotée, et qui explique en partie les dimensions exceptionnelles des bâtiments présents dans l'enclos, notamment sa grange.

La léproserie cesse de recevoir des dons dès la fin du $\mathrm{XIII}^{\mathrm{e}}$ siècle. Vers la fin du XIV e siècle, le domaine n'est plus exploité par faire-valoir direct et les terres sont mises à bail. Face au recul de la lèpre à la fin du Moyen Âge, Saint-Lazare recueille désormais les pauvres et des malades victimes d'autres

1. Je remercie tout particulièrement Jean-Marc Fémolant, directeur du Service archéologique de Beauvais, qui est à l'initiative de cette étude et qui a fortement contribué à la réalisation financière et matérielle de ce travail.

2. LEBLOND 1922, p. 1.

3. Extrait du $2^{\text {nd }}$ cartulaire de l'eglise de Beauvais, Coll. Troussures, pièce justificative no 2, cité dans Edelman 1979, p. 9.

4. AD Oise, Arch. Hospit. St.-Lazare, FI et B35.

5. Edelman 1979, p. 29-49. affections de la peau ${ }^{6}$. La léproserie tombe en commende au début $\mathrm{du} \mathrm{Xvi}^{\mathrm{e}}$ siècle et les religieux qui assuraient la gestion des biens et les soins aux malades quittent définitivement SaintLazare, entraînant un relâchement de la discipline, la location des terres et la décadence de l'établissement. Cependant, devant la recrudescence de la peste dans la seconde moitié du $\mathrm{XVI}^{\mathrm{e}}$ siècle et au début du XVII ${ }^{\mathrm{e}}$ siècle, de nouveaux bâtiments furent construits au sein de l'enclos pour recevoir l'afflux de malades. Enfin, en 1658, Saint-Lazare est rattachée au Bureau des pauvres de Beauvais ${ }^{7}$.

Devenue Bien National à la Révolution, Saint-Lazare perd définitivement sa vocation hospitalière. Elle est vendue en plusieurs lots et convertie en exploitation agricole jusqu'en 1920 où elle est rachetée par une entreprise de produits chimiques. Classée sur la liste des Monuments historiques en 1862, déclassée en 1887 puis inscrite de nouveau en 1939, l’ancienne léproserie Saint-Lazare est rachetée par la ville de Beauvais en 1988 pour être transformée en centre à vocation culturelle.

\section{LA LÉPROSERIE}

Située à un kilomètre au sud-est de Beauvais, au bord de l'ancienne voie menant à Paris, la léproserie se présente dans un enclos polygonal de 2,5 hectares, fermé par un mur de deux mètres de haut, avec une porterie donnant directement sur la voie (fig. 1). L'espace intérieur est compartimenté en trois zones distinctes, aux fonctions spécifiques : la première consacrée aux lépreux, la seconde destinée à la gestion administrative et religieuse de l'établissement et la troisième à vocation agricole.

L'espace réservé aux lépreux, au sud, était pourvu d'une succession de loges accolées à l'enceinte et formant une cour fermée. À l'est, subsistent les ruines des bâtiments destinés aux pestiférés, édifiés au $\mathrm{XVI}^{\mathrm{e}}$ siècle ou au début du XVII ${ }^{\mathrm{e}}$ siècle et circonscrits dans une cour fermée. L'espace dédié aux activités administratives, communautaires et religieuses occupe la place centrale de l'établissement, face à la porterie. Il comprend une église, pourvue d'un cimetière au sud, un grand logis, dont la charpente est datée de 1272 d, voué aux activités et à l'hébergement des clercs, un réfectoire, disparu au début du siècle et enfin, un troisième grand édifice à deux nefs, pourvu d'un niveau sur cave, en majeure partie détruit. Le troisième espace, au nord-ouest de l'enclos, consacré aux activités agricoles, comprenait une bergerie, en partie conservée, une étable, l'habitation du fermier dans l'angle ouest de l'enclos, un colombier ainsi qu'une grange (fig. 2). Cet espace était occupé au nord-est par des vignes, attestées dès le XIII ${ }^{\text {e }}$ siècle et destinées à l'approvisionnement en vin des lépreux.

Ce dernier ensemble de bâtiments, assimilable aux trois autres granges de l'établissement, constituait le pôle d'exploitation du domaine agricole attenant à la léproserie. En effet, pour le $\mathrm{XIII}^{\mathrm{e}}$ siècle, le cartulaire témoigne de donations, de legs et de rachats de terres cultivables, de prés, de bois et de vignes situées à proximité immédiate de la léproserie et dans les localités avoisinantes. Comme pour les autres granges, ces rachats et les échanges de terres avaient pour objectif de constituer un domaine homogène et sans enclave, concentré,

6. LEBLOND, 1926, p. 30.

7. BnF, Arch. Nat., S 4827. 


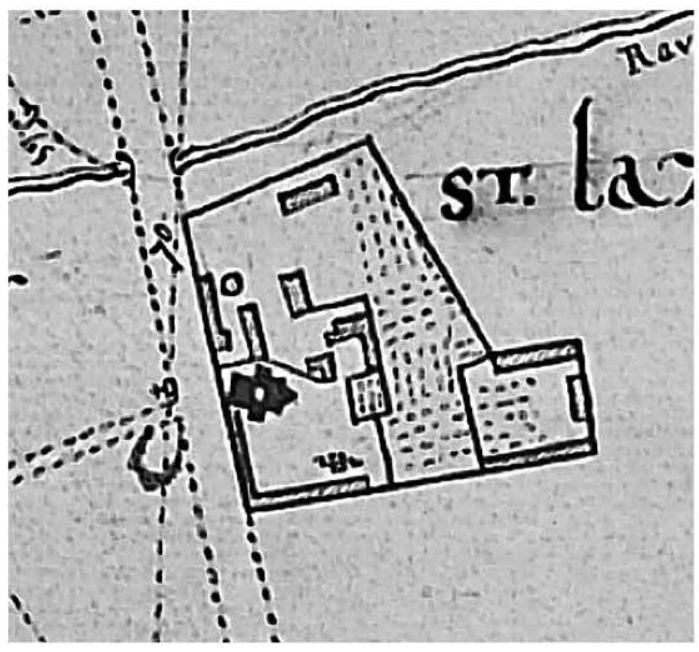

1. Loges des lépreux

2. Léproserie moderne

3. Eglise

4. Porterie

5. Logis

6. Réfectoire

7. Bâtiment à deux nefs

8. Bergerie

9. Pigeonnier

10. Grange

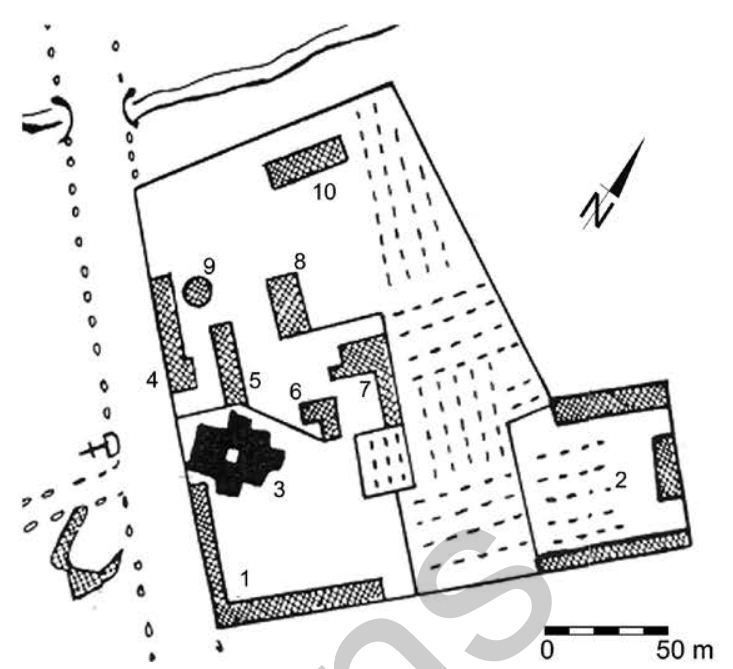

Fig. 1 Plan de la léproserie en 1782 (Archives départementales de L’Oise - Carte générale des fiefs et seigneurie du champ Jean Leblond appartenant à l'évêque de Beauvais, 1782, procès-verbal d'arpentage, Allonne, 1Cp3/1)

ici, autour de la léproserie pour des raisons pratiques de mises en culture, comme faciliter l'assolement triennal, limiter la circulation des charrois, le coût du transport et, à terme, optimiser la productivité du domaine.

La grange de Saint-Lazare devait être destinée au stockage des céréales du domaine attenant. Récoltés à la faucille lors des moissons d'été, fin juin pour le seigle et jusqu'en août parfois pour le froment ou l'avoine, les blés étaient engrangés sous forme de gerbes bottelées et chargées par empilement. Le déchargement des chariots dans la grange se faisait par le bas-côté sud dans lequel ils circulaient en sens unique d'un pignon à l'autre. À l'époque moderne, le remplissage de la grange montait à plus de $10 \mathrm{~m}$ de hauteur comme le prouvent des brins de blé retrouvés coincés dans des assemblages au sommet des fermes et de nombreux graffiti sur les bois en partie haute de la charpente ${ }^{8}$. Le battage au fléau de ces gerbes de blé se faisait dans un second temps, quelques semaines après la moisson, lorsque la main-d'œuvre était libérée par la fin des récoltes. Cette opération était généralement faite sur une aire de battage, au sol battu, à l'entrée la plus ventilée de la grange pour séparer les grains de la paille broyée.

\section{LE BÂTI MAÇONNÉ}

Orientée nord-est/sud-ouest, la grange présente un plan rectangulaire de 47,20 m de long sur 21,95 m de large, hors œuvre des murs, et une superficie intérieure de $762 \mathrm{~m}^{2}$ (fig. 3 et 4). Elle est subdivisée en une nef centrale et deux bas-côtés par deux rangées de neuf grandes arcades. D'épais contreforts saillants, à retraites talutées avec larmier, épaulent les murs pignons au droit des arcades et aux angles du bâtiment. Les pignons

8. Les graffiti des $\mathrm{XVIII}^{\mathrm{e}}$-XXe siècles correspondent à des noms associés à une date avec, parfois, le témoignage d'une bonne récolte de blé. Les plus originaux concernent les signatures et matricules de militaires de la Grande Guerre. Des rosaces gravées au compas, vraisemblablement du XIII siècle, se rencontrent en grand nombre sur les murs, au-dessus de l'extrados des grandes arcades, au centre d'une pile, au-dessus du ressaut, et à une hauteur correspondant aux niveaux d'échafaudage d'après les trous de boulins.

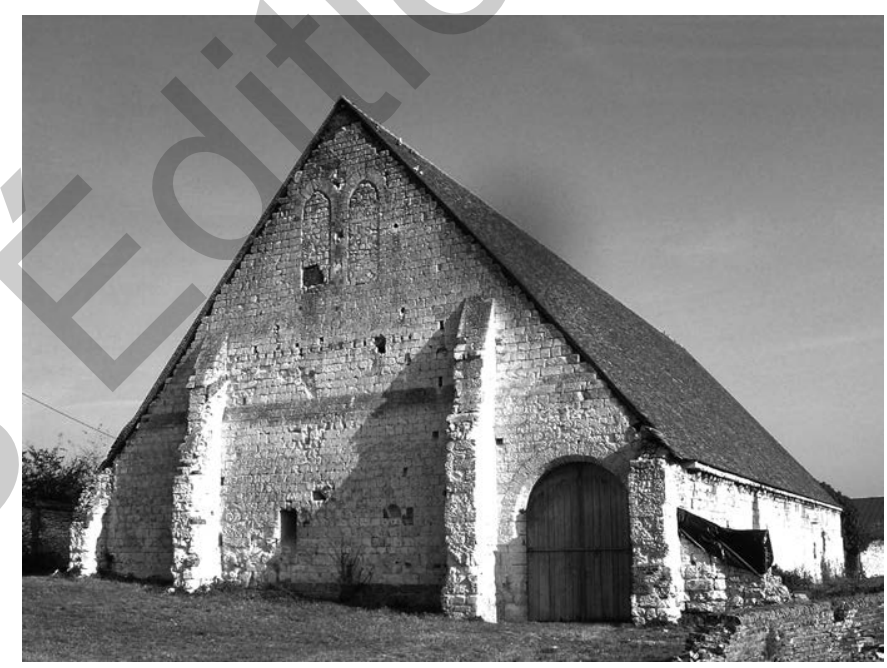

Fig. 2 Pignon ouest de la grange (cl. F. E.).

s'amincissent en hauteur au tiers inférieur de leur élévation, comme les gouttereaux par un petit retrait extérieur. Chaque pignon est percé d'une porte charretière qui s'ouvre sur un unique couloir de circulation dans le bas-côté sud dont le sol est encore en terre battue à la chaux pour faciliter le charroi. Une niche est aménagée dans le mur gouttereau sud, le long de ce couloir, en face la $6^{\text {e }}$ pile ouest. L'éclairage est assuré par deux lancettes en tiers point, au sommet de chaque pignon (fig. 2 et 3). Si des traces d'un cloisonnement tardif sont visibles dans leurs embrasures, il ne semble pas que ces baies aient été fermées à l'origine pour permettre justement la ventilation de la grange.

Construite sur une déclivité de $4 \%$ du terrain, la grange a été en partie excavée pour obtenir un sol intérieur horizontal, ce qui explique les nombreuses reprises, liées aux infiltrations, de la base intérieure du gouttereau nord, située à $1,25 \mathrm{~m}$ sous le sol extérieur. Édifiées en assises horizontales, les maçonneries sont en moyen appareillage de pierres calcaires, taillées par layage ou par brettelure, selon quatre hauteurs d'assise distinctes, marquées de I à IIII au ciseau dans l'ordre croissant : 

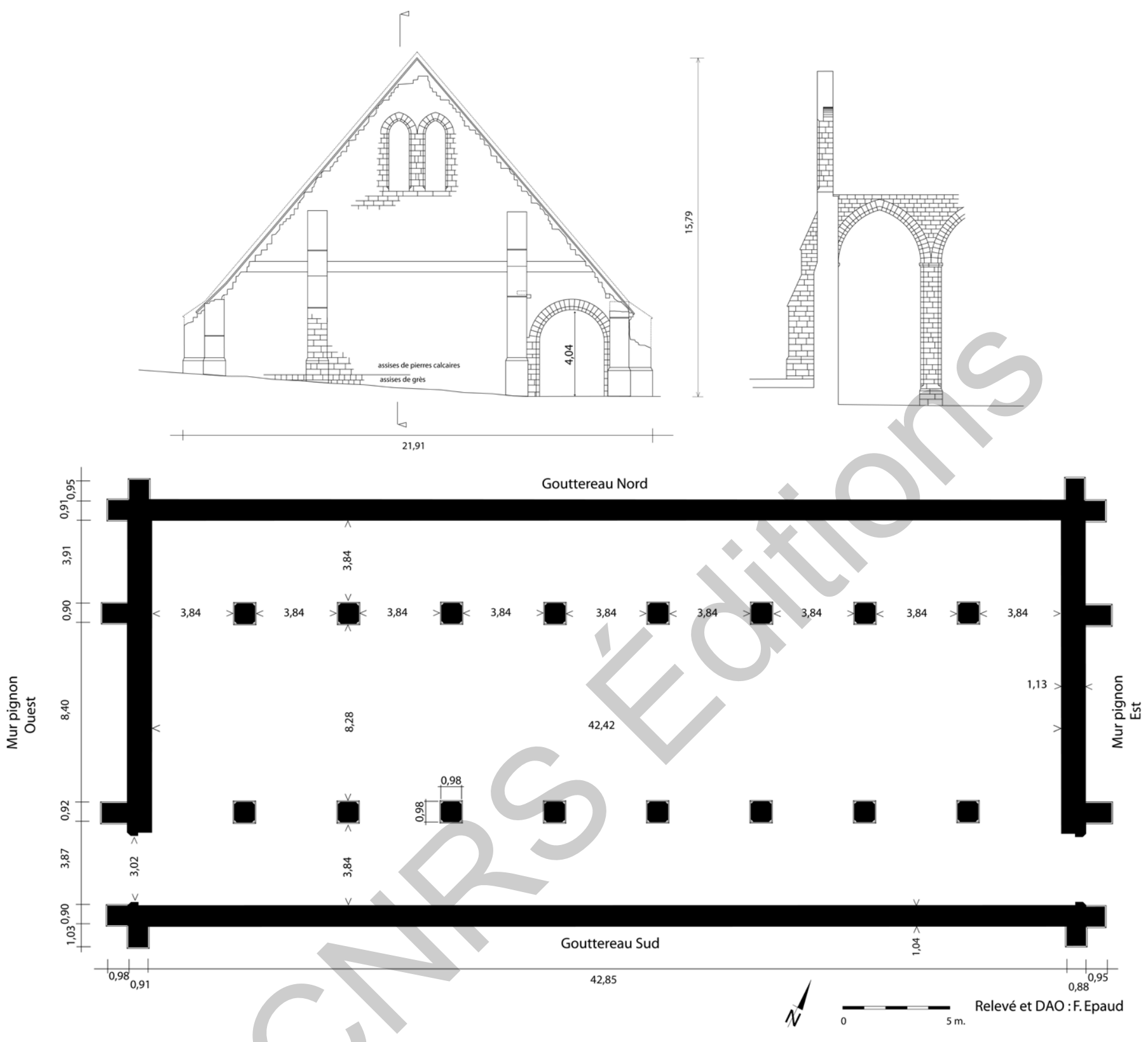

Fig. 3 Plan de la grange, élévations et coupe transversale du mur pignon ouest.

$18,3 \mathrm{~cm}, 21,3 \mathrm{~cm}, 24 \mathrm{~cm}$ et $27 \mathrm{~cm}$. Sur le pourtour extérieur de l'édifice, la base des murs comporte, au contact du sol, deux à trois assises de grès qui suivent le dénivelé du terrain et qui servent à protéger les maçonneries des remontées capillaires. Au sommet des gouttereaux, une corniche est soutenue par une suite de modillons moulurés. Les solins de toiture appareillés qui couraient en tête des murs pignons ont disparu.

Les neuf grandes arcades, disposées en deux files parallèles, retombent sur des piliers de section carrée (fig. 4). Ces piles ont un empattement à leur base, de hauteur variable selon les piles, et des arêtes chanfreinées sur toute leur hauteur jusqu'à un ressaut à la retombée des arcades. Celles-ci ont un profil d'arc brisé surbaissé et présentent un unique rouleau chanfreiné aux arêtes. Sous les grandes arcades et aussi en parties hautes des murs pignons, subsistent des restes d'un enduit de couleur beige-ocre. Vu la répartition de cet enduit sur les murs et son état de conservation, il est très vraisemblable Qu'il fut réalisé lors de l'édification de la grange et qu'il recouvrait l'ensemble des maçonneries intérieures.

Sur le parement intérieur du gouttereau nord, près du pignon est, sont conservés les tracés d'une épure gravée dans la pierre avec une fine pointe métallique, représentant quatre axes rectilignes entrecroisés et trois arcs d'ouvertures distinctes (fig. 5). Son usure et son positionnement à hauteur d'homme, entre les trous de boulins du mur, nous incitent à penser que ces tracés sont contemporains du chantier du XIII ${ }^{\mathrm{e}}$ siècle. En effet, d'après la restitution des tracés directeurs, l'arc le plus ouvert concorde précisément au profil des grandes arcades, et le trait horizontal, compris entre les points de rencontre des deux traits verticaux, correspond au rayon du cercle qui sert à tracer cet arc. Ce tracé est basé sur une mesure étalon de deux pieds à $0,64 \mathrm{~m}(2 \times$ $0,32 \mathrm{~m}$ ) qui se retrouve dans l'ouverture de l'arcade à sa base 


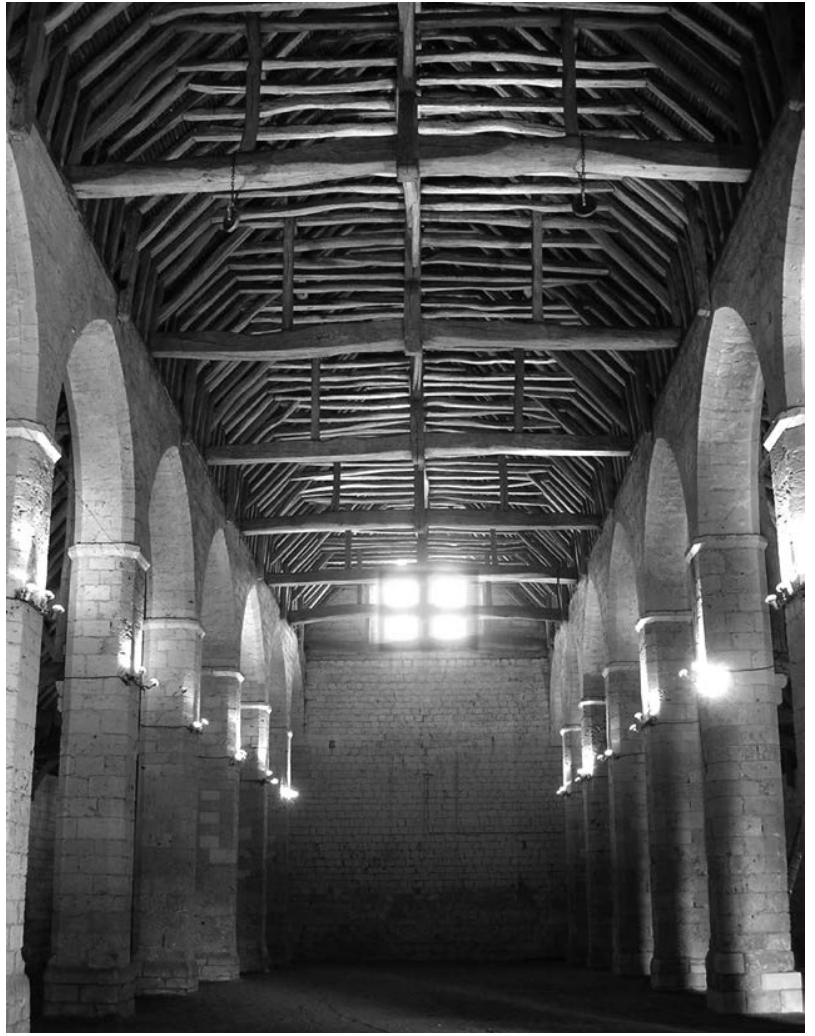

Fig. 4 Vue du vaisseau central de la grange (cl. F. E.).

$(3,84 \mathrm{~m}=6 \times 0,64 \mathrm{~m})$, soit 6 unités ou 12 pieds, comme dans le tracé de l'arc $(2,56 \mathrm{~m}=4 \times 0,64 \mathrm{~m})$, soit 4 unités ou 8 pieds dont le centre du cercle est placé aux deux tiers de l'ouverture de l'arcade. Si ces tracés correspondent bien à l'épure des grandes arcades, les autres arcs gravés ainsi que le positionnement des axes rectilignes demeurent encore incompris.

Les deux portes charretières qui s'ouvrent sur le couloir du bas-côté sud sont couvertes chacune d'un arc en plein cintre avec, face interne, un linteau d'origine ${ }^{9}$ en chêne ancré aux extrémités dans les maçonneries, sous un second arc segmentaire rehaussé. Ces linteaux ${ }^{10}$ recevaient, à chaque bout, dans une entaille cylindrique, en vis-à-vis d'une autre taillée dans le seuil en grès, l'arbre tournant de deux ventaux qui s'ouvraient vers l'intérieur. À l'extérieur, au-dessus des deux portes, des logements contenant des bois, datés par dendrochronologie ${ }^{11}$ de l'origine de la construction, témoignent d'une charpente en appentis qui servait d'auvent de protection pour chaque entrée (fig. 6). Cette charpente comprenait un chevronnage qui s'appuyait sur un couple de sablières basses, soutenues par deux consoles dont le chapeau ${ }^{12}$ était ancré dans le mur, et une sablière haute qui était portée par deux corbeaux en bois également insérés dans le mur. Un larmier maçonné marquait la naissance de cette toiture.

9. Le Digol et Bernard 2006.

10. Section : $25 \times 26 \mathrm{~cm}$.

11. Le Digol et Bernard 2006.

12. Pièce horizontale.
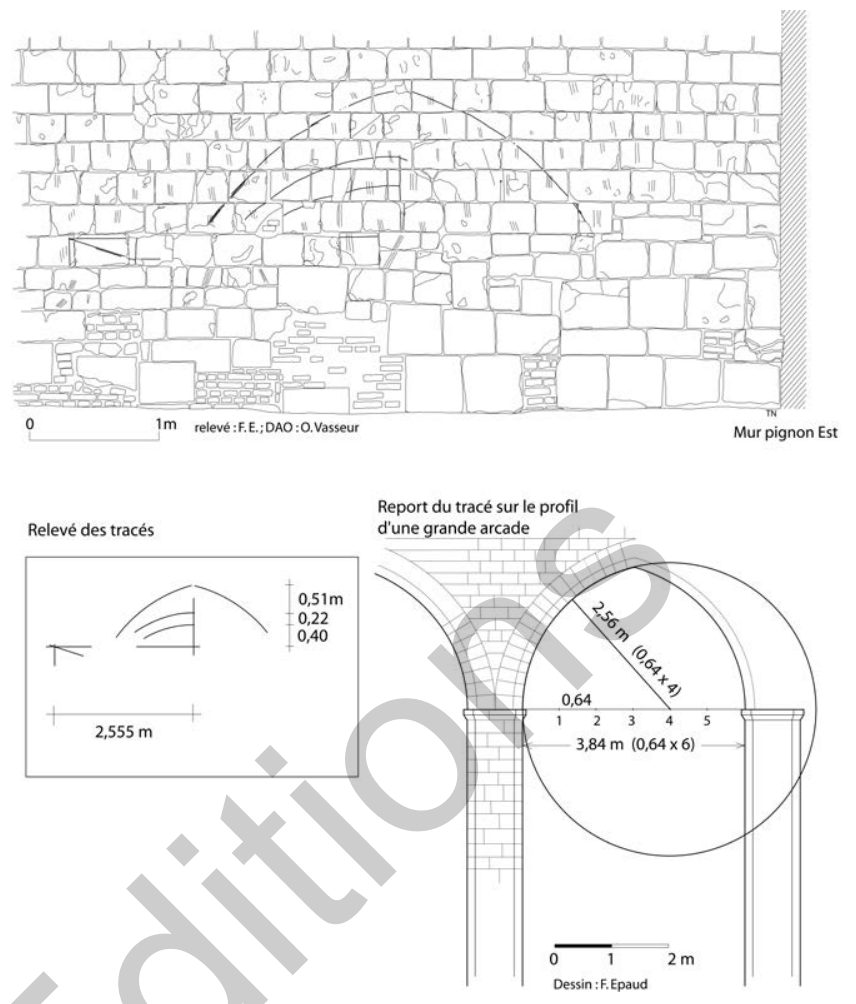

Fig. 5 Relevé du mur gouttereau nord, face intérieure, avec restitution des tracés.

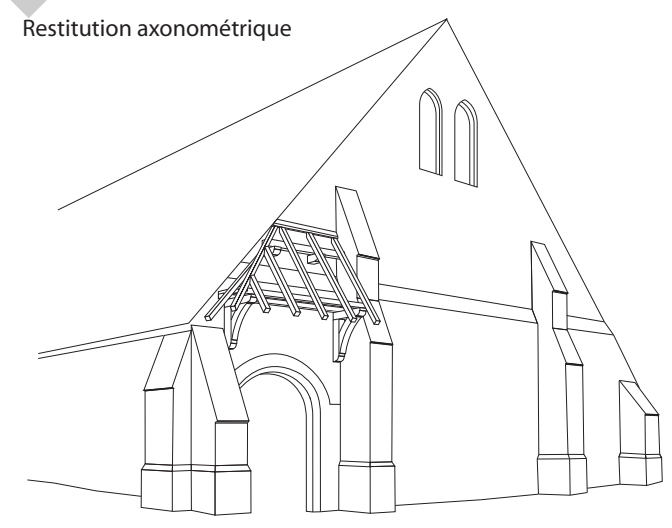

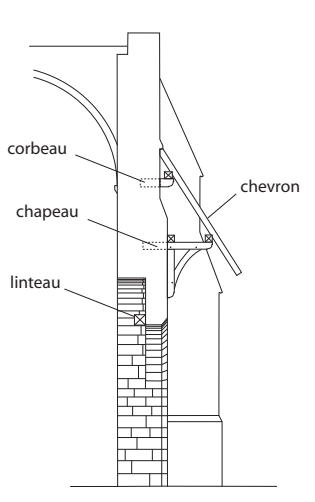

Coupe longitudinale

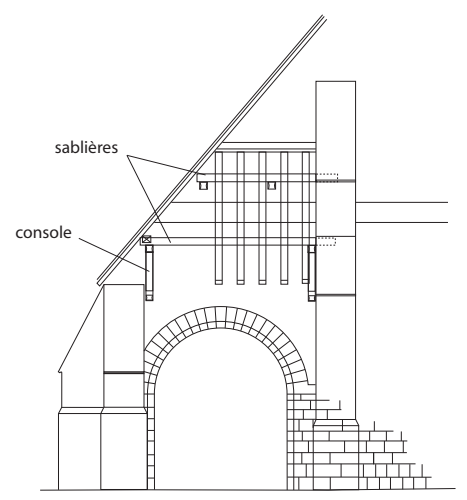

Elévation de l'auvent, face est

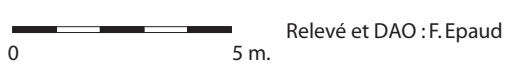

Fig. 6 Restitution de l'auvent du pignon oriental. 


\section{LA CHARPENTE}

L’ensemble de la structure se trouve dans un très bon état de conservation. Seules les deux dernières travées ouest du bas-côté sud ont connu des remaniements à la fin du Moyen Âge du fait probable de l'adjonction d'un édifice contre la toiture, dans le prolongement de la bergerie. Les restaurations menées à partir des années 1970 par l'architecte en chef des Monuments historiques Jean-Pierre Paquet n'ont touché que les sablières externes des bas-côtés et quelques pieds de fermes occidentales.

Réalisée par le laboratoire Dendrotech, l'étude dendrochronologique a déterminé, sur la base de 30 échantillons prélevés dans l'ensemble de la charpente, une unique phase d'abattage durant l'automne-hiver 1219-1220, plus précisément entre le mois d'octobre 1219 et le mois de février $1220^{13}$.

\subsection{LES BOIS}

Tous les bois de cette charpente sont des bois de brin ${ }^{14}$ en chêne équarris à la doloire. Aucune trace de scie n'a été observée, même en about de pièce comme les extrémités des blochets. Sur de nombreux bois, on distingue parfois des traces de coups de hache espacés de 30 à $40 \mathrm{~cm}$, larges, peu profonds et inclinés par rapport à l'axe du bois. Ces traces témoignent d'un ébauchage ou dégrossissage préliminaire qui consiste à réaliser des encoches rapprochées pour faciliter l'équarrissage dans le sens du fil du bois ${ }^{15}$.

Cette charpente a été réalisée avec des bois de profils homogènes. Ces derniers présentent tous de nombreuses courbures, des flaches ${ }^{16}$ importantes et une grande variation de la section pour chaque type de pièce. Il semble donc que les charpentiers n'ont pas eu le choix dans la sélection des bois d'œuvre pour ce chantier et qu'ils ont dû se contenter des seuls bois qui leur étaient disponibles, et ce, malgré les moyens visiblement conséquents affectés à la construction de cette grange. Ce cas de figure peut donc correspondre à une coupe à blanc d'une parcelle de futaie au peuplement homogène, et non à un abattage par furetage ${ }^{17}$ qui suppose une sélection des arbres sur une plus grande superficie, avec donc des sujets au profil de meilleure qualité.

Si l'utilisation de bois courbes est généralement problématique pour réaliser une charpente, ici, les charpentiers ont su en tirer le meilleur parti. Les bois les moins courbes ont été utilisés pour les sablières et les poinçons, par nécessité de planéité de leurs quatre faces. Les bois pour les chevrons ont été orientés de façon à ce que la face la plus droite serve au lattis, au détriment de leurs flancs qui présentent de fortes courbures. Idem pour les pannes et les liernes dont la face la plus droite sert à porter les entraits retroussés. Partout ailleurs, la courbure est mise à profit pour améliorer la résistance de la pièce comme les entraits, convexes en face supérieure pour lutter contre sa flexion ou

13. Le Digol et Bernard 2006.

14. Bois qui conserve le cœur de l'arbre.

15. Cette technique est encore pratiquée en Tchéquie et en Roumanie (BHAHA, JANÀK et RUZICKA 2008).

16. Arrondi d'une arête provenant de la surface naturelle du tronc de l'arbre que l'équarrissage n'a pas pu corriger.

17. Coupe ponctuelle d'un bel arbre isolé et adapté au but recherché. les arbalétriers, pour résister à la compression des pannes. La courbure des poteaux des bas-côtés, placés contre les piles, leur permet d'éviter le ressaut maçonné de ces dernières.

Trois types de grumes ont été utilisés pour la réalisation de cette charpente. Le premier correspond aux gros bois destinés aux entraits, de $10,30 \mathrm{~m}$ de long. Pour une section médiane de $0,40 \times 0,40 \mathrm{~m}$, on estime leur pied à $0,50 \times 0,50 \mathrm{~m}$, soit un diamètre de $0,70 \mathrm{~m}$ environ en pied, pour une hauteur de fût de $11 \mathrm{~m}$ minimum. Le second concerne les pièces de fortes sections comprises entre $0,25 \times 0,25 \mathrm{~m}$ et $0,30 \times 0,30 \mathrm{~m}$ comme les poteaux, les entraits et les arbalétriers des demi-fermes. Leur section définit des grumes d'un diamètre moyen de $0,38 \mathrm{~m}$ en pied pour une hauteur minimum de $7 \mathrm{~m}$. Enfin, la troisième mesure s'applique à tous les autres bois, d'une section inférieure à $20 \times 18 \mathrm{~cm}$. Parmi ceux-là, les bois de faible section comme les entretoises de $0,11 \times 0,11 \mathrm{~m}$ correspondent aux extrémités des bois de 0,15 $\times 0,16 \mathrm{~m}$ qui, eux-mêmes, se situent dans le prolongement des pièces de $0,20 \times 0,18 \mathrm{~m}$. La grande majorité de ces bois sont issus de grumes d'un diamètre compris entre $0,27 \mathrm{~cm}$ et $0,15 \mathrm{~m}$, avec une moyenne de $0,24 \mathrm{~m}$, pour une longueur minimale de $9 \mathrm{~m}$.

Tableau de la consommation de grumes dans la charpente

\begin{tabular}{|l|l|l|l|}
\hline $\begin{array}{l}\text { Mesure des } \\
\text { grumes } \\
\text { consommées } \\
\text { (diamètre au pied } \\
\text { et longueur) }\end{array}$ & Pièces & $\begin{array}{l}\text { Quantité } \\
\text { de grumes } \\
\text { consommées }\end{array}$ & Proportion \\
\hline $0,70 \mathrm{~m} / 11 \mathrm{~m}$ & Entraits & 10 & $1 \%$ \\
\hline $0,38 \mathrm{~m} / 7 \mathrm{~m}$ & $\begin{array}{l}\text { Poinçons, } \\
\text { entraits } \\
\text { et arbalé- } \\
\text { triers des } \\
\text { demi-fermes }\end{array}$ & 58 & $8 \%$ \\
\hline $0,24 \mathrm{~m} / 9 \mathrm{~m}$ & $\begin{array}{l}\text { Chevrons, } \\
\text { raidisseurs et } \\
\text { pièces longi- } \\
\text { tudinales }\end{array}$ & 692 & $91 \%$ \\
\cline { 3 - 5 } & & Total : 760 & $100 \%$ \\
\cline { 2 - 4 } & &
\end{tabular}

En quantifiant les grumes utilisées pour chaque type de ferme, selon les trois mesures identifiées, et en additionnant les longueurs de pièces de section voisine jusqu'à obtenir la longueur maximale, on estime, pour la construction de toute la charpente, une consommation de 760 grumes, dont $91 \%$ de faible diamètre et de grande longueur, et $1 \%$ de fort diamètre.

Les analyses dendrochronologiques ont confirmé, par ailleurs, que les bois de faible diamètre ont des fortes croissances et qu'ils avoisinent une cinquantaine d'années, tandis que ceux de forte section (entraits) ont des croissances plus lentes pour des bois deux fois plus âgés ${ }^{18}$. Cette forte proportion de bois jeunes, minces et de grande longueur, abattus en une seule saison, confirmerait un approvisionnement par une coupe massive dans une forêt exploitée en taillis-sous-futaie, avec de vieux semenciers de fort diamètre mis en réserve. L'homogénéité physiologique de ces bois, abattus en une seule

18. Le Digol et Bernard 2006. 
campagne, tendrait aussi à démontrer que cette coupe à blanc s'est opérée dans une unique forêt homogène. Cependant, si tous ces bois ont été abattus en une seule coupe, leur exécution et leur montage ont vraisemblablement nécessité un à deux ans de travaux, vu les dimensions de l'ouvrage, mais à partir du même lot de bois. Cette mise en chantier a été consécutive à l'abattage comme le révèle la déformation des marques d'assemblage ou des trous de cheville par des fentes de séchage. Ces modes de consommations sont similaires à ceux observés dans les charpentes des cathédrales de Rouen, Lisieux, Bayeux et aussi de l'abbaye de Bonport (Eure) ${ }^{19}$, toutes de la première moitié du XIII ${ }^{\mathrm{e}}$ siècle, ce qui supposerait, à l'échelle d'un vaste territoire, l'existence d'une logique raisonnée des approvisionnements et d'une gestion maîtrisée des massifs forestiers.

\subsection{LA STRUCTURE}

La charpente à chevrons-formant-fermes ${ }^{20}$ de la nef comprend 80 fermes de trois types distincts : les fermes principales, assises au droit des piles, les fermes intermédiaires situées en milieu de travée et les fermes secondaires (fig. 7 à 11). Leur répartition définit neuf travées, chacune constituée d'une ferme principale, d'une ferme intermédiaire et de six ou sept fermes secondaires. Leur distribution s'articule précisément avec la trame des grandes arcades de façon à répartir les charges de la charpente sur les piles. Cette disposition témoigne donc d'une concertation des maîtres maçons et charpentiers pour la conception et la cotation des structures maçonnées et charpentées.

\section{Plan et cotations}

Comme les arcades retombent dans les murs pignons sur des culots intégrés et non sur des piles, entraînant un raccourcissement de la travée de l'épaisseur d'une demi-pile, les première et dernière travées de charpente sont plus courtes de $0,50 \mathrm{~m}$ que les autres (fig. 8). Les deux fermes principales situées aux extrémités de la charpente sont disposées dans une retraite de 0,20 m des murs pignons, ce qui place leur axe précisément dans le plan vertical de ces murs. De ce fait, l'emplacement des piles, toutes espacées de 4,82 m d'axe en axe, définit une répartition régulière des fermes principales, secondaires et intermédiaires selon un entraxe de $0,537 \mathrm{~m}$ précisément pour l'ensemble de la charpente.

\section{Les sablières et le système des cales}

Les fermes s'appuient sur deux cours continus de larges sablières reliées entre elles tous les 3 à $4 \mathrm{~m}$ par des entretoises assemblées par des mi-bois en demi-queue d'aronde. D'une longueur variant de 2,60 m à 10,13 m, avec une moyenne comprise entre 4 et $6 \mathrm{~m}$, les sablières s'aboutent entre elles par des entures biaises crantées, fixées par une cheville verticale. Ces entures ne sont jamais placées sous un blochet ou un entrait, pour ne

19. ÉPaud 2007, p. 15.

20. Structure dans laquelle chaque chevron est assemblé au chevron du versant opposé par des raidisseurs pour former une ferme.

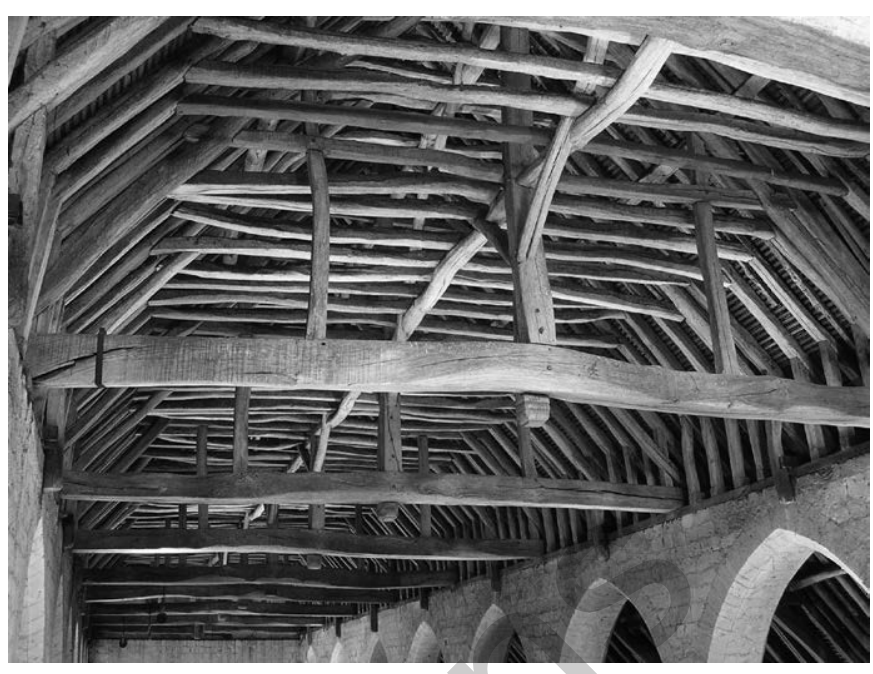

Fig. 7 Charpente du vaisseau principal (cl. F. E.).

pas fragiliser les assemblages, et sont réparties de façon à ce que les sablières internes et externes soient disposées en quinconce, selon leur longueur, afin d'éviter le vis-à-vis de leurs entures et renforcer ainsi leur stabilité face aux poussées latérales des fermes secondaires.

Comme les sablières du bas-côté nord sur les trois travées orientales, les sablières sud de la nef, sur les quatre travées orientales, reposent sur des cales en bois, réparties uniformément sous elles, notamment sous chaque enture (fig. 12). Ces cales sont soit courtes, disposées sous une seule sablière, soit longues, passant à la fois sous les sablières interne et externe. Dans ce cas, elles viennent en butée au nu du mur par un crochet taillé en quart-de-rond et chanfreiné aux arêtes. Au-delà de la quatrième travée orientale, elles disparaissent et les sablières passent d'un niveau surélevé de $0,3 \mathrm{~m}$ au plat du mur en fléchissant sur deux mètres. La présence de ces cales peut résulter d'une correction de l'assise des fermes ou à un problème de raccord survenu au cours du levage entre les chevrons de ce versant et ceux du bas-côté sud auxquels ils sont assemblés.

\section{Les fermes principales}

Disposées au droit des piles et contre les murs pignons, les fermes principales sont constituées chacune d'un entrait, d'un poinçon, de deux poteaux latéraux, d'un couple d'arbalétriers, d'un couple de chevrons inclinés à $49,3^{\circ}$ et raidis par deux faux entraits et deux jambes de force avec, en complément, deux entretoises qui servent à porter les pannes (fig. 11). Long de $10,30 \mathrm{~m}$ avec une portée de 8,36 m dans œuvre des murs, l'entrait présente une section médiane de $0,40 \times 0,40 \mathrm{~m}$ et de $0,32 \times 0,32 \mathrm{~m}$ aux extrémités, ce qui suppose un équarrissage forcé de son extrémité côté houppier ${ }^{21}$ pour réduire le poids de l'entrait et équilibrer les charges sur les murs. Ces entraits sont assemblés, en sous-face, aux sablières par des entailles à queue-d'aronde, orientées de façon à lutter contre la flexion de l'entrait et l'écartement des sablières lié aux poussées latérales des autres fermes (fig. 13, en haut à gauche). Tous ces entraits

21. Ensemble des branches d'un arbre, en partie haute. 


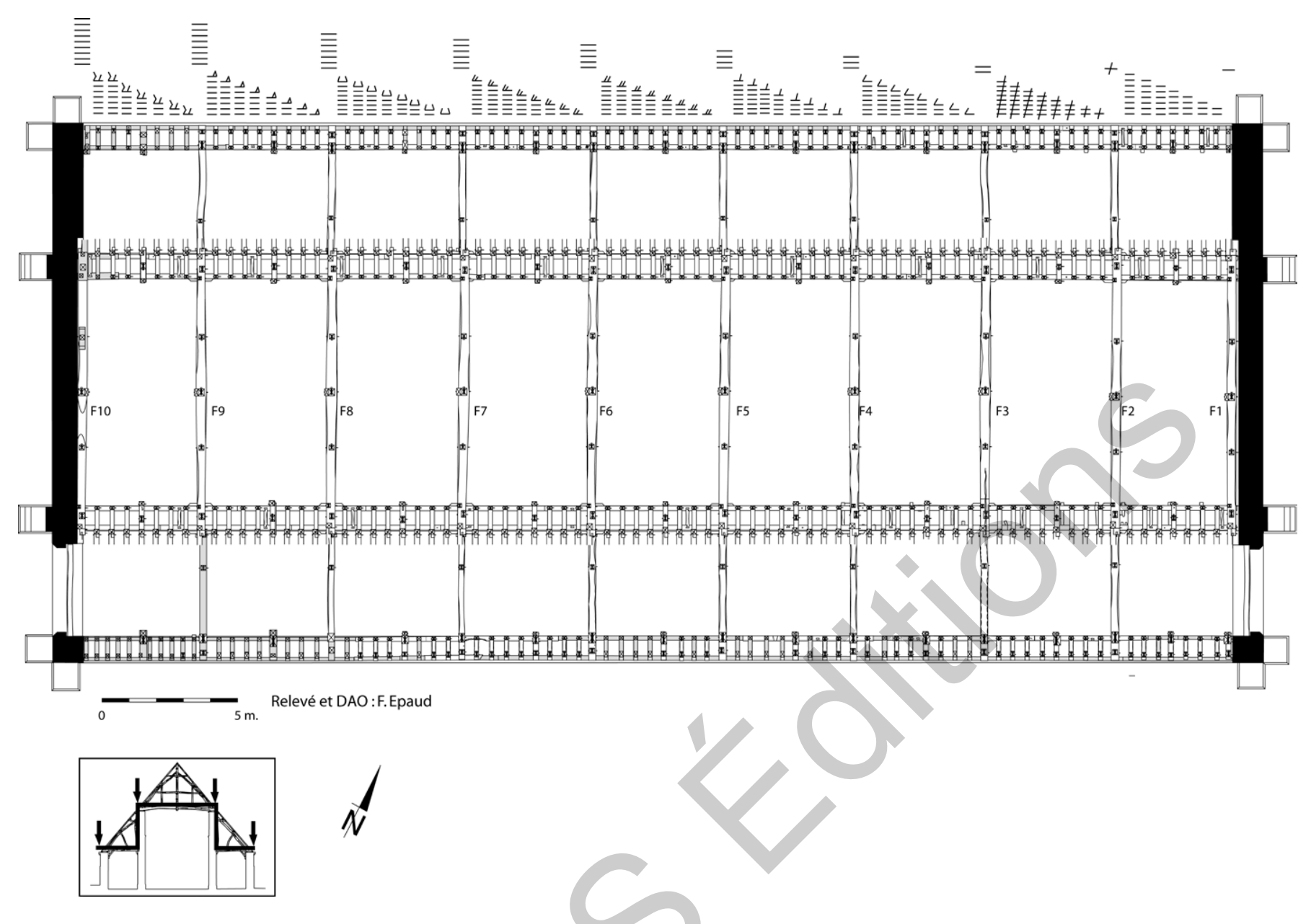

Fig. 8 Plan au niveau des sablières.

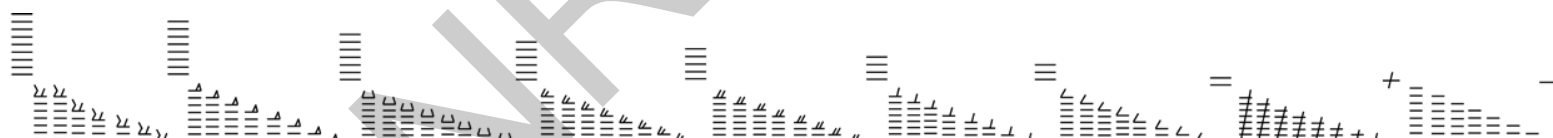

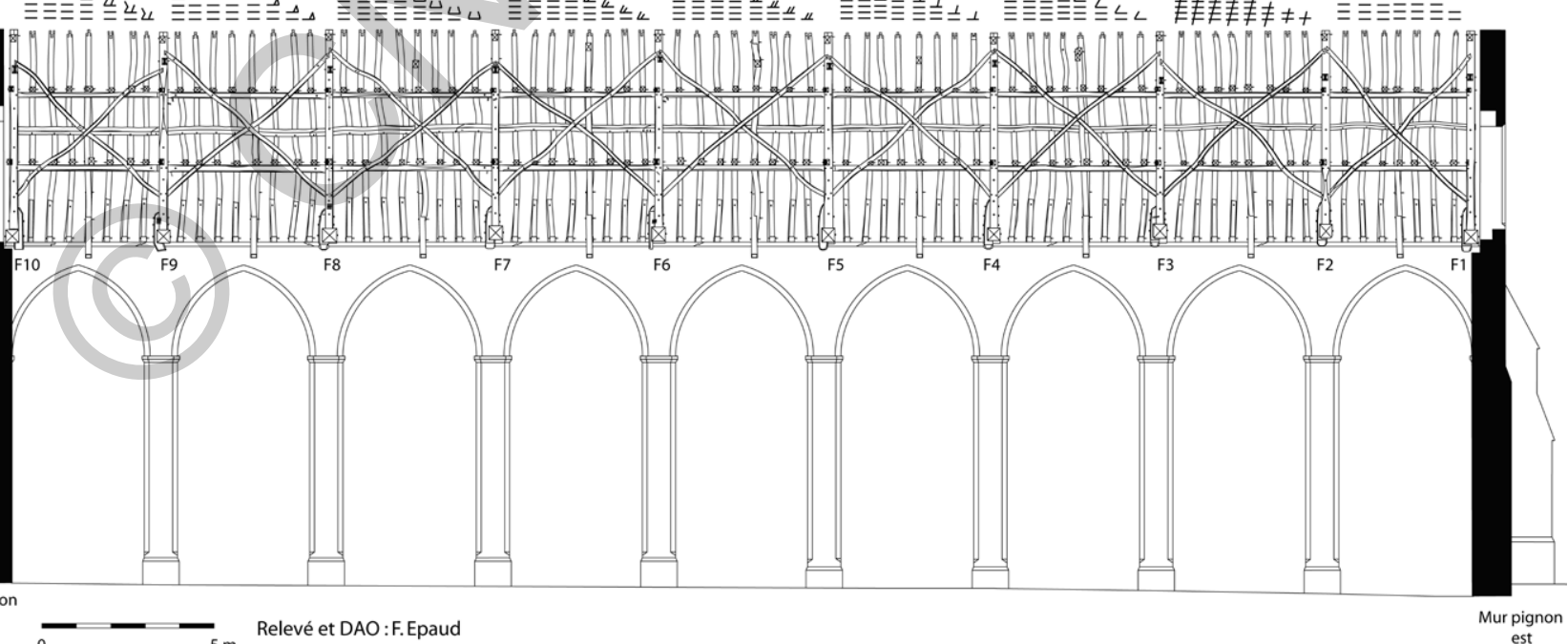

ouest $\quad-{ }_{5 \mathrm{~m}}$. Relevé et DAO:F.Epaud

Fig. 9 Coupe longitudinale ouest-est du vaisseau principal. 


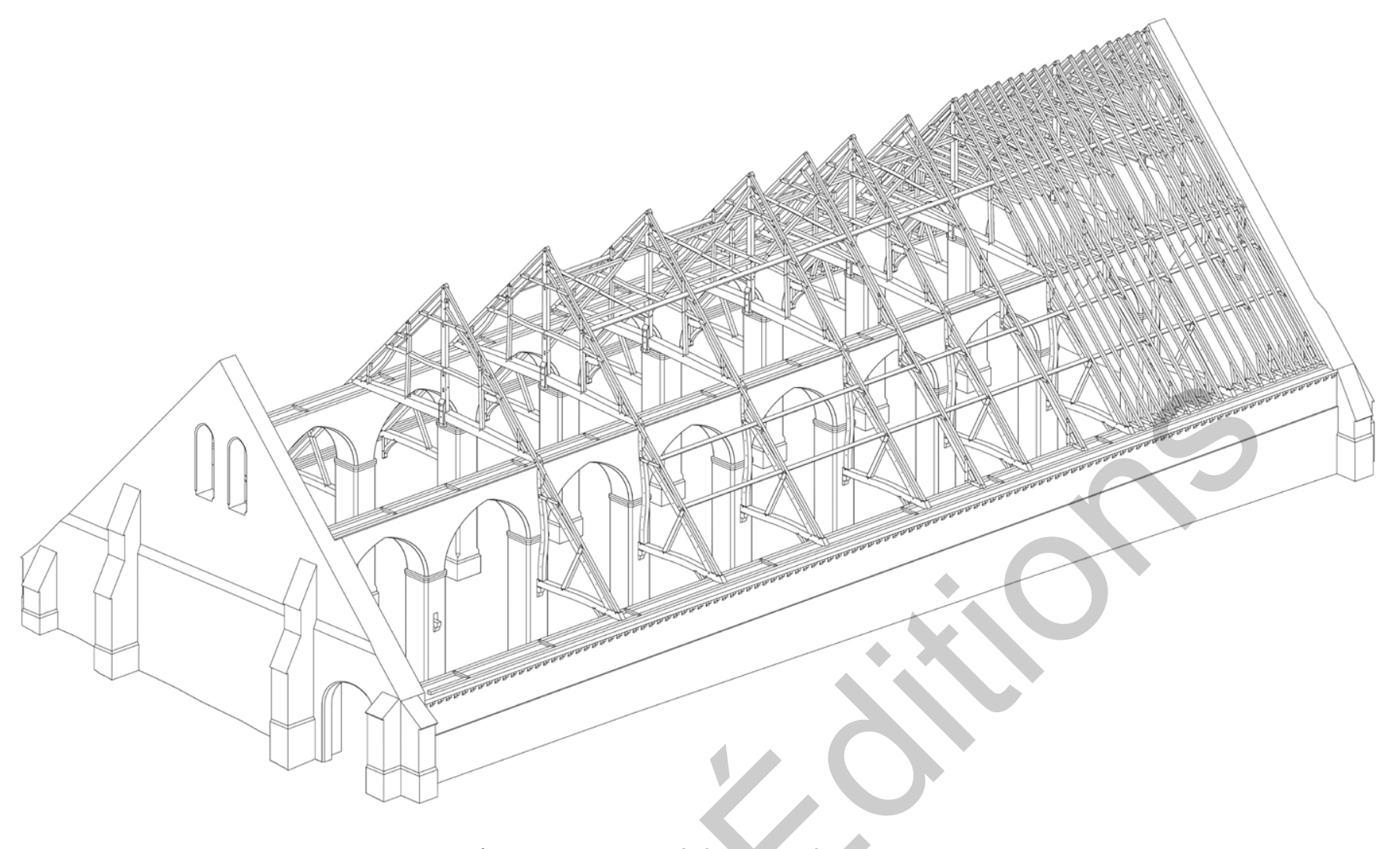

Fig. 10 Axonométrie de la grange (dessin : F. E.).

ont leur courbure orientée vers le haut pour lutter contre leur propre flexion.

Longs de $8,70 \mathrm{~m}$, les chevrons ont une section moyenne de $0,15 \times 0,18 \mathrm{~m}$ à la base et de $0,13 \times 0,16 \mathrm{~m}$ en tête, avec la face la plus large destinée à la fixation du lattis. Ils sont assemblés en tête dans le poinçon par un mi-bois flanqué d'un talon triangulaire qui vient en butée dans le poinçon, participant ainsi à sa suspension (fig. 13, au centre). Ils sont raidis par deux faux entraits ${ }^{22}$, assemblés par tenon-mortaise, et une jambe de force, par un embrèvement biais, chevillé par l'extérieur. En pied, le chevron franchit l'entrait par un mi-bois pourvu d'un talon qui lui assure une butée sur l'entrait, et vient s'abouter au chevron du bas-côté par une enture biaise chevillée (fig. 13, en bas).

Parallèlement aux chevrons, deux arbalétriers viennent s'assembler en pied dans l'entrait et en tête dans le poinçon par tenon-mortaise. Leur fonction est de porter à dévers ${ }^{23}$ les pannes et de réceptionner leurs charges sur la ferme principale en induisant une compression en tête du poinçon. Pour cette raison, ils ont une forte section ${ }^{24}$ avec une courbure orientée vers le haut pour mieux résister aux compressions des pannes. Si ces arbalétriers servent à porter les pannes en les orientant à dévers, ce sont surtout les poteaux latéraux et leur entretoise, fixés par mi-bois au chevron, qui assurent leur calage (fig. 14). Ils sont

22. $0,17 \times 0,15 \mathrm{~m}$ pour le premier faux entrait inférieur et $0,15 \times 0,13 \mathrm{~m}$ pour le second.

23. Position dans laquelle la pièce a ses faces latérales non verticales et perpendiculaires au chevron.

24. $0,18 \times 0,17 \mathrm{~m}$ en pied et $0,16 \times 0,15 \mathrm{~m}$ en tête. souvent désaxés par rapport au plan de la ferme pour éviter d'entailler trop profondément, par leur mi-bois, l'arbalétrier, le chevron et le premier faux entrait. Assemblés en tête au chevron et à l'arbalétrier, ces poteaux ne s'appuient pas sur l'entrait, mais le soutiennent comme en témoigne leur assemblage de pied, par tenon-mortaise en demi-queue d'aronde, bloqué par une petite cale, dite rossignol, qui assure la suspension de ce dernier aux poteaux.

Le poinçon, de forte section ${ }^{25}$, est tenu en tête par les chevrons et les arbalétriers qui lui permettent de suspendre en pied l'entrait selon le principe de la triangulation grâce, là aussi, à un tenon-mortaise en demi-queue d'aronde, bloqué par un rossignol (fig. 15). Cette traction est renforcée par une suspente, c'est-à-dire une pièce de bois destinée à suspendre ici l'entrait, assemblée au poinçon et à l'entrait par des adents imbriqués en forme de trapèze, et maintenue par quatre fortes chevilles. La base de la suspente est moulurée avec des arêtes chanfreinées.

Dans les bas-côtés nord et sud, les demi-fermes principales, situées dans le prolongement des fermes principales (fig. 11 et 16), sont constituées d'un entrait, d'un poteau, d'une contrefiche, d'un chevron doublé d'un arbalétrier, d'une entretoise et d'une console, sous l'entrait. La disposition de ces pièces est très ingénieuse, car certaines ont une double fonction. La contrefiche et l'entretoise, assemblées par un mi-bois à demi-queued'aronde au chevron, portent les deux cours de pannes, mais ils assurent également le raidissement de l'arbalétrier et du chevron. Le poteau, qui s'assemble en tête dans l'entrait de la ferme principale par un tenon-mortaise, lui-même recouvert

25. $0,25 \times 0,24 \mathrm{~m}$ à la base et $0,22 \times 0,21 \mathrm{~m}$ en tête. 


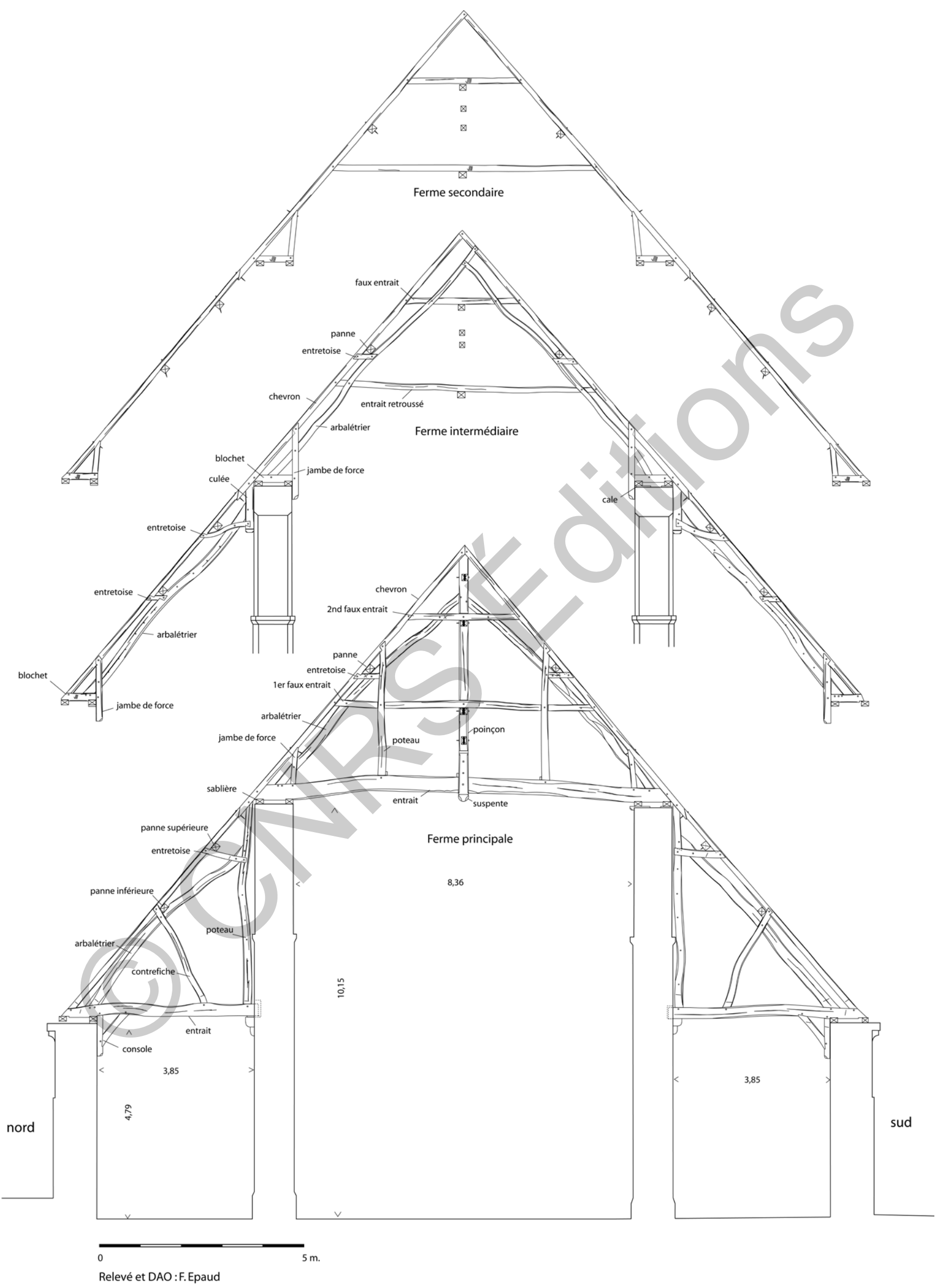

Fig. 11 Ferme principale 2, ferme intermédiaire de la 3e travée, et ferme secondaire de la 3e travée, face ouest. 


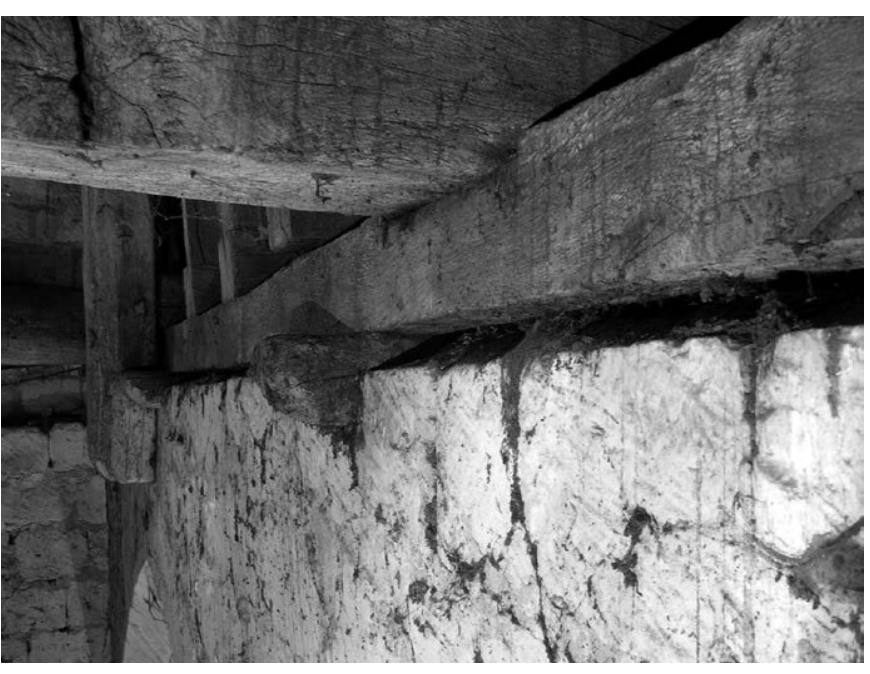

Fig. 12 Cale avec crochet sous les sablières sud (cl. F. E.).

par le mi-bois du chevron (fig. 13, en bas), réceptionne l'arbalétrier, mais aussi l'entretoise en récupérant ainsi une partie des charges transmises par les pannes, d'où sa forte section ${ }^{26}$. L'arbalétrier porte les pannes, mais il renforce également la résistance des raidisseurs du chevron auxquels il est assemblé par mi-bois. L'entrait, quant à lui, travaille, d'une part, contre les poussées latérales imposées par l'arbalétrier et le chevron, d'où la console en sous-face qui vient en butée contre le mur. D’autre part, il lutte contre les lourdes charges compressives transmises par le poteau et la contrefiche, d'où, là aussi, sa forte section. Cet entrait est ancré dans le pilier, au droit de culots taillés en quart-de-rond, tandis que l'autre extrémité est assemblée aux sablières par un mi-bois à queue et demi-queue-d'aronde.

La courbure naturelle de ces bois est mise à profit dans chaque circonstance, avec un bombement tourné de façon à mieux lutter contre les charges de compression, que ce soit pour l'entrait, l'arbalétrier ou la contrefiche. La torsion du poteau est quant à elle orientée pour éviter le ressaut maçonné de la pile. Des cales sont très souvent présentes pour compenser ces courbures comme entre la panne et l'arbalétrier, ou sous l'entrait, dans la cavité de la pile. D'autres cales sont parfois placées sur l'entretoise, sous la panne supérieure qui est posée à dévers, et donc uniquement sur son arête inférieure, pour garantir sa stabilité.

\section{Les fermes intermédiaires}

Situées en milieu de travée, les fermes intermédiaires ont pour fonction de soulager le cours des pannes à mi-portée (fig. 11). Elles sont constituées d'un couple de chevrons, assemblés en tête par enfourchement, raidi par un entrait retroussé et un faux entrait, de deux arbalétriers, deux jambes de force et deux blochets. Du fait de l'absence d'entrait, la ferme a tendance à s'écarter à sa base en exerçant des poussées latérales sur les maçonneries. Ces poussées sont contenues par le premier entrait retroussé qui travaille en traction, et par une jambe de force de forte section qui se prolonge au-delà du blochet

26. $0,22 \times 0,22 \mathrm{~m}$ en pied et $0,18 \times 0,20 \mathrm{~m}$ en tête.
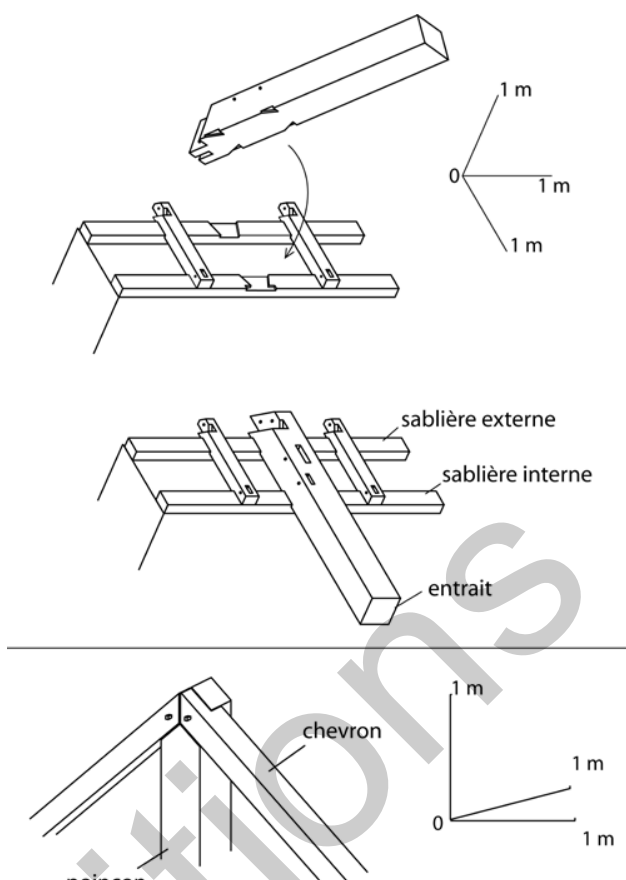

poinçon
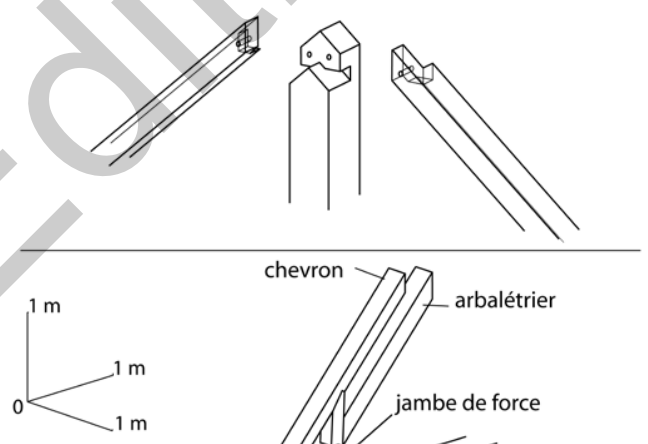

chevron arbalétrier

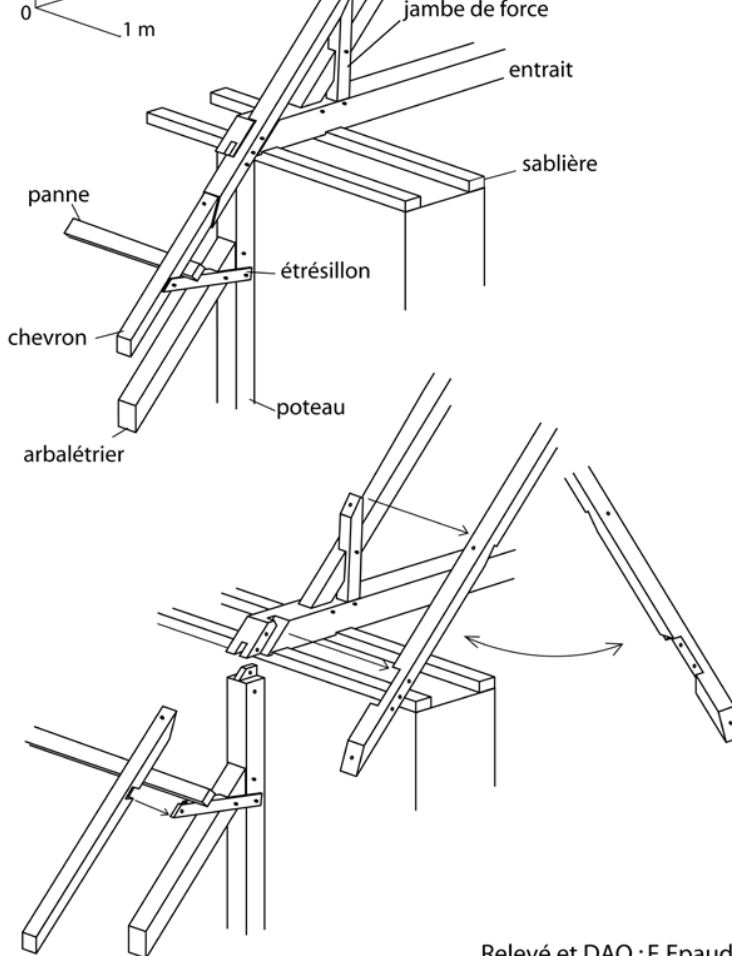

Relevé et DAO :F. Epaud

Fig. 13 Ferme principale : assemblages de l'entrait aux sablières (en haut), des chevrons en tête du poinçon (au centre) et du pied (en bas). 


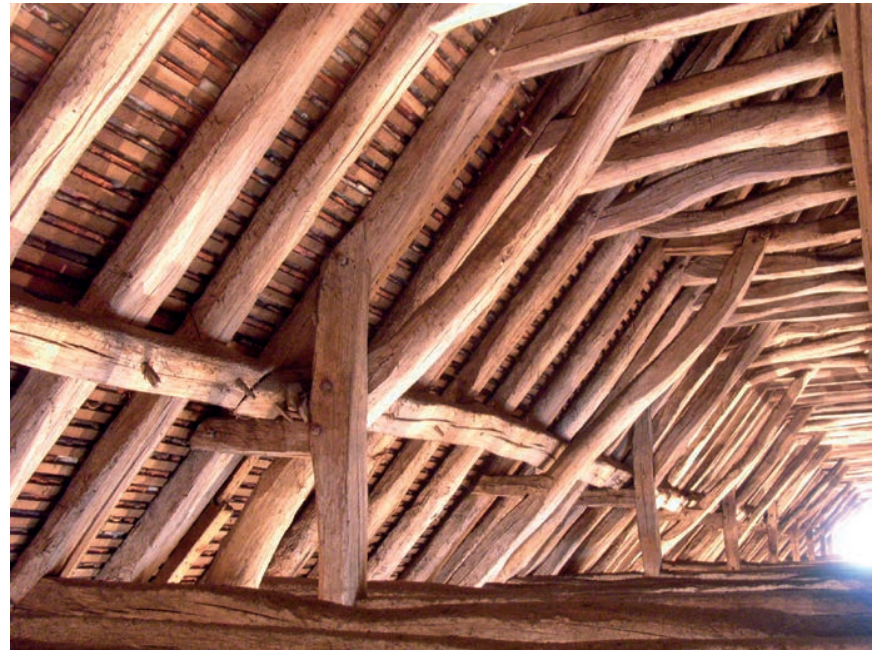

Fig. 14 Calage des pannes sur l'arbalétrier par le poteau latéral et son entretoise, assemblés à mi-bois au chevron (cl. F. E.).

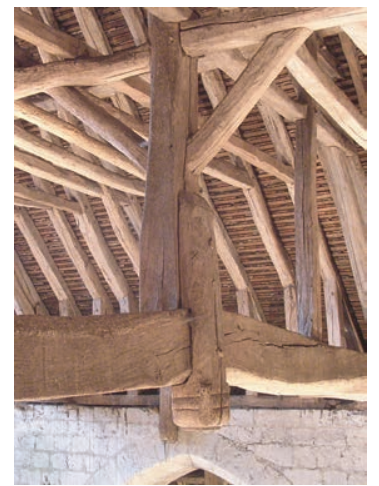

Cl. F. Epaud

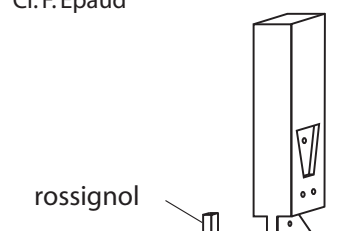

rossignol $>$ Tा

o

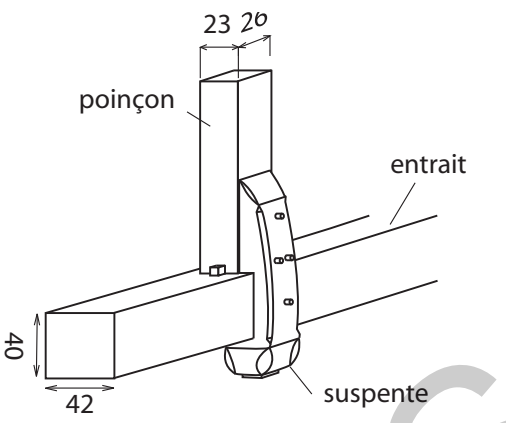

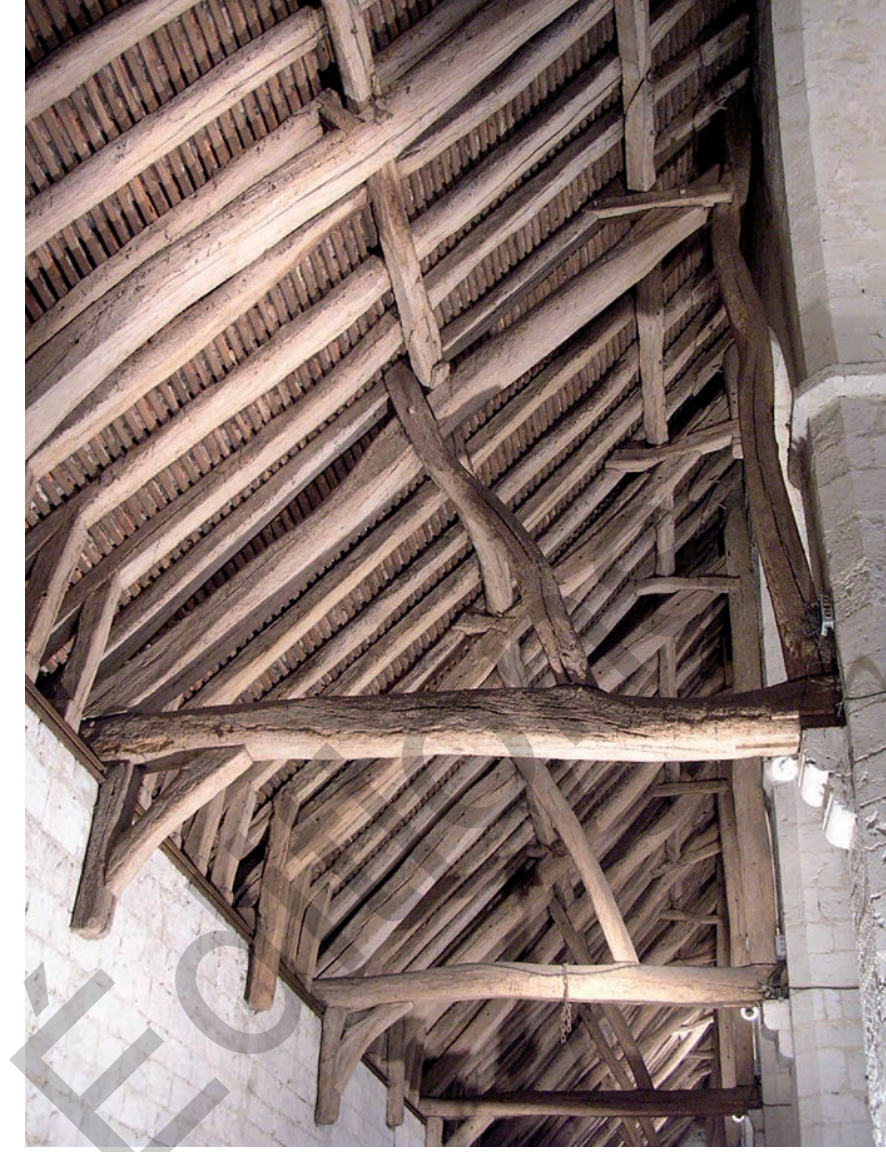

Fig. 16 Demi-ferme principale 8 du bas-côté nord (cl. F. E.).

Les chevrons ${ }^{27}$ s'assemblent dans le prolongement de ceux des bas-côtés par une enture biaise chevillée, et au blochet et à la tête de la culée du bas-côté par un mi-bois particulièrement complexe (fig. 17 à droite). Comme pour les fermes principales, le mi-bois du chevron est pourvu d'un talon en équerre qui prend appui dans le blochet, pour lutter contre les poussées latérales. Par ailleurs, le chevron participe à la suspension de la culée, également par un talon qui y pénètre, perpendiculairement à son axe, pour assurer sa retenue. Cet assemblage ingénieux par sa taille et sa conception remplit donc plusieurs fonctions cumulées en traction et en compression.

Sur le même principe que les fermes principales, deux arbalétriers sont intégrés à la ferme, parallèlement aux chevrons et assemblés en tête par mi-bois, pour porter les cours de pannes par l'entremise d'une entretoise assemblée à mi-bois en demi-queue d'aronde. Leur fonction consiste à soulager le cours des pannes en milieu de travée et à renforcer la structure de la ferme, comme une attelle, en doublant tous les assemblages des raidisseurs. C'est une mise en tension conjointe de l'ensemble des pièces qui permet de compenser la reprise des charges des pannes.

Dans les bas-côtés, les demi-fermes prolongent les fermes intermédiaires de la nef, en milieu de travée et aussi contre les murs pignons en raison de l'impossibilité d'y placer des demi-fermes principales, faute de pile pour ancrer l'entrait. Destinées à soulager le cours des pannes en milieu de portée, 


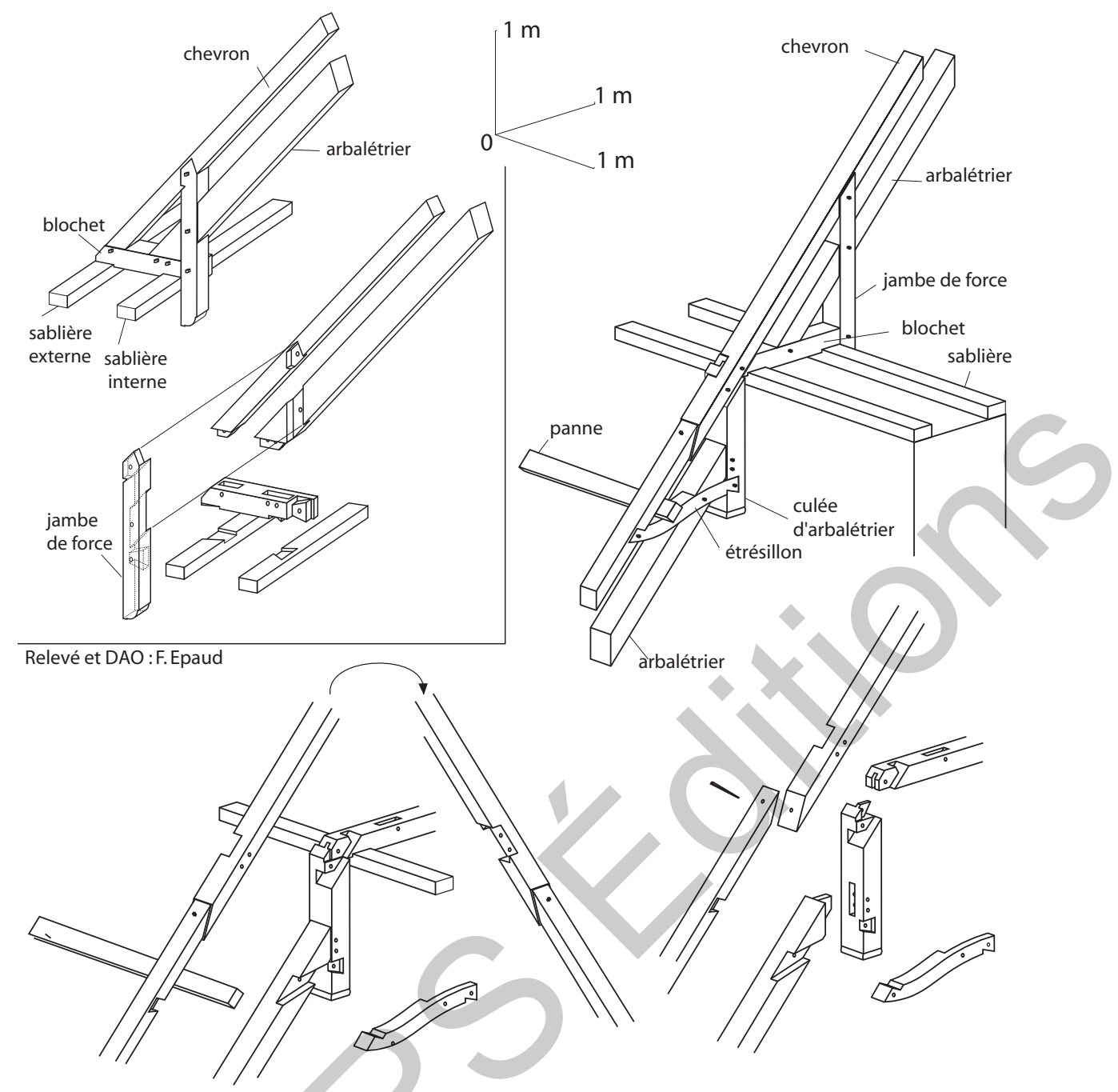

Fig. 17 Ferme intermédiaire: assemblages de la jambe de force en butée contre le mur (en haut à gauche) et de la jonction de la tête d'une demi-ferme.

elles résistent à la compression des pannes et luttent contre de fortes poussées latérales, du fait de l’absence d'entrait retroussé. La solution adoptée est particulièrement astucieuse puisqu'elle utilise le principe de la mise en tension de l'arbalétrier, couplé à deux dispositifs complexes de compensation de charges en pied et en tête de la demi-ferme. Le nœud d'assemblages du chevron, du blochet, de la jambe de force et de l'arbalétrier constitue un cadenas indéformable, en mesure d'absorber ces poussées (fig. 17 en haut à gauche). La jambe de force, assemblée par mi-bois, se prolonge au-delà du blochet pour prendre appui contre le mur et travailler en butée contre lui. Elle assure ainsi le blocage de ce cadenas et la mise en tension des autres pièces. En tête de la demi-ferme, le dispositif permet de retenir l'arbalétrier grâce à un jeu compensatoire des tensions entre le chevron, la culée et l'entretoise qui porte la panne supérieure et bloque le dispositif par des mi-bois (fig. 17 à droite). L'arbalétrier prend appui sur la culée suspendue au blochet par un tenon-mortaise en demi-queue d'aronde, et au chevron par un mi-bois pourvu d'un talon perpendiculaire à l'axe de la culée. Venant en butée contre cette culée, l'arbalétrier peut ainsi être mis en tension et supporter les deux cours de pannes, soutenues chacune par une entretoise assemblée à mi-bois. Réagissant comme une attelle, comme dans les fermes de la nef, larbalétrier absorbe la flexion du chevron et compense, grâce à ses dispositifs de pied et de tête, les poussées latérales de la demi-ferme. On note, par ailleurs, que pour soutenir la panne supérieure, l'entretoise ne pouvait être taillée dans un bois droit, mais nécessairement dans un bois courbe, en forme de $S$ ou fortement incurvé, pour éviter une trop forte inclinaison de l'entretoise et donc des assemblages peu résistants.

\section{Les fermes secondaires}

Au nombre de sept par travée, et de six dans la première et la dernière travée, les fermes secondaires sont constituées d'un couple de chevrons, de plus faible section ${ }^{28}$ que les précédents, assemblés en tête par enfourchement, raidis par un entrait retroussé, un faux entrait, et un couple de jambes de force

28. $0,13 \times 0,18 \mathrm{~m}$ à la base et $0,12 \times 0,15 \mathrm{~m}$ en tête. 
assemblées par embrèvement biais et chevillé depuis l'intérieur (fig. 11). Le blochet se fixe aux sablières par un mi-bois à queue et à demi-queue-d'aronde pour mieux résister aux poussées latérales. Comme pour les fermes précédentes, le chevron s'assemble au blochet par un mi-bois avec un talon perpendiculaire à l'axe du chevron, pour éviter son glissement.

Dans les bas-côtés, le prolongement des fermes secondaires se limite à un simple chevron, assemblé en tête au chevron de la nef par une enture biaise et, en pied, à un blochet par un tenon-mortaise et à une jambe de force par un embrèvement biais. Les blochets sont quant à eux assemblés par mi-bois aux sablières. Là aussi, la face la plus large du chevron est destinée à la fixation du lattis. Leur chevillage à la jambe de force et aux deux cours de pannes est réalisé depuis leur face supérieure.

\section{Le dispositif longitudinal}

Toute la charpente est traversée longitudinalement par un dispositif axial fixé aux poinçons des fermes principales (fig. 9). Il est constitué de deux cours de liernes horizontales, tenonnées aux poinçons, et disposées sous le niveau des entraits retroussés et des faux entraits, auxquels ils ne sont pas chevillés (fig. 18). Dans chaque travée, les liernes sont raidies par une croisée de grandes écharpes assemblées aux poinçons, en tête, par un mi-bois à demi-queue-d'aronde ou avec ergot, selon l'inclinaison des bois, sur la face sud et, en pied, par tenon-mortaise. Du fait de la torsion naturelle des bois, le franchissement des écharpes à travers le réseau des faux entraits et des entraits retroussés a entraîné de nombreuses retailles des bois pour permettre leur passage, au risque de les fragiliser.

Ces liernes servent à supporter les entraits des fermes secondaires et contribuent ainsi, à limiter leur tassement et les poussées latérales sur les murs. Elles récupèrent leurs charges pour les reporter sur les fermes principales, au droit des piles, libérant ainsi les grandes arcades de ces contraintes. Ces charges, transmises aux poinçons sont compensées en partie par la triangulation. Par ailleurs, les grandes écharpes dont la vocation est de raidir les liernes, assurent indirectement le contreventement des fermes principales.

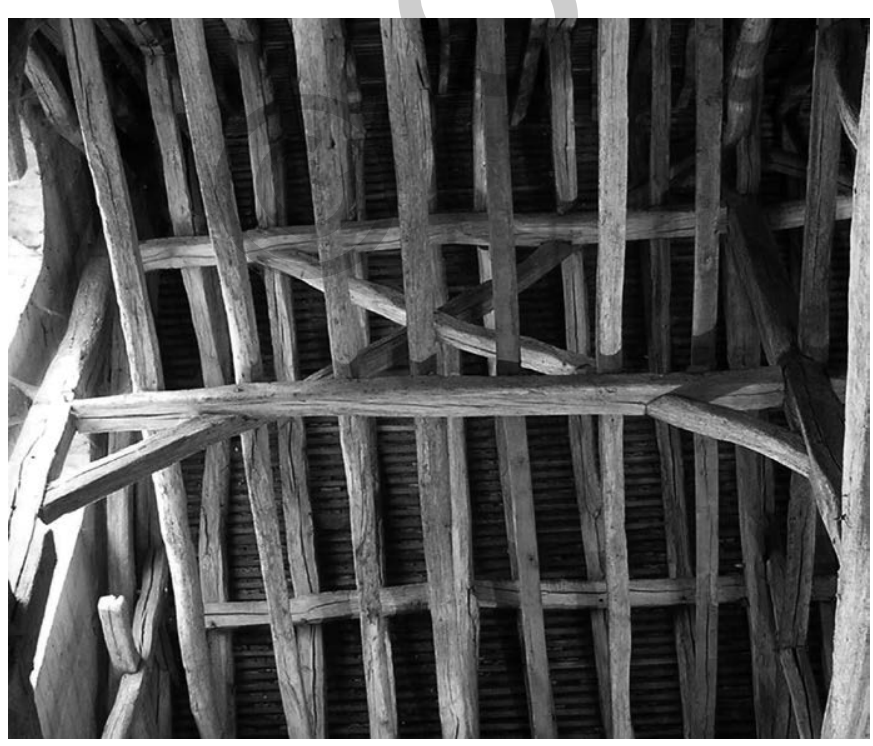

Fig. 18 Dispositif longitudinal de la première travée ouest (cl. F. E.).
Ce dispositif est renforcé dans son action par le cours des pannes qui supportent les chevrons auxquels elles sont chevillées, depuis l'extérieur. Ces pannes sont aboutées entre elles par une enture biaise chevillée à proximité de chaque ferme principale. Les charges du chevronnage sont véhiculées par ces pannes jusqu'aux fermes principales et, à mi-portée, aux fermes intermédiaires qui sont assises au droit de l'extrados des arcades, c'est-à-dire au point de leur plus forte résistance. Ces pannes participent donc, au même titre que les liernes et les écharpes, au transfert des charges de la travée vers les points des supports maçonnés les plus forts, afin de libérer les arcades des poussées latérales des fermes secondaires.

\subsection{LE MARQUAGE DES BOIS}

Sur les fermes, toutes les marques sont inscrites à la rainette ${ }^{29}$, sur la face ouest des bois, la face d'assemblage des mi-bois, et couvrent en général toute la largeur de la face (fig. 8). Elles sont localisées en pied de pièce pour les éléments verticaux ou obliques et, en milieu de pièce ou bien à un emplacement suffisamment large et dépourvu de flache pour les pièces horizontales, sauf pour les entraits principaux qui, eux, sont marqués à leur extrémité sud. Il s'agit de marques de pièce et non de marques d'assemblage puisque chaque pièce n'en possède qu'une seule, et que celle-ci n'est pas reportée sur l'autre pièce au droit de l'assemblage, à quelques exceptions près. Aucune contremarque n'a été relevée pour distinguer les pièces du versant nord de celles du versant sud.

Le plan de marquage est cohérent et s'applique aussi bien aux fermes de la nef qu'aux demi-fermes des bas-côtés. Les fermes principales ont une numérotation continue de bâtonnets qui évolue de l'est vers l'ouest, partant de I sur la première ferme orientale, sautant la seconde ferme marquée d'une croix, reprenant à II sur la troisième ferme pour terminer à IIIIIIII (8) sur la dernière. Cette série présente donc une anomalie au début où il semble bien que la seconde ferme a été marquée I, par méprise, comme la première et que, pour corriger cette erreur, ce I a été barré pour former une croix. En effet, sur cette seconde ferme, plusieurs bois marqués I ont été oubliés et se mélangent avec des bois inscrits d'une croix.

Indépendamment des fermes principales, chaque travée a une numérotation propre. Sans distinguer les fermes intermédiaires des fermes secondaires, ni celles des bas-côtés, chacune des travées présente une série continue, de l'est vers l'ouest, de I à IIIIIIII (8), sauf pour les première et dernière travées qui se terminent à IIIIIII (7). Ces séries se distinguent par un signe particulier rajouté au premier bâtonnet de la marque, à l'exception de la première série. Ces signes correspondent soit à un trait barrant la marque, un crochet, une ou deux «langues de vipère", placées différemment ou encore plus original, sur la dernière travée, plusieurs crochets.

Outre un plan de marquage cohérent pour l'ensemble des fermes de la charpente, l'écriture de ces marques est identique pour toutes ces fermes : même type de rainette, largeur de la marque, emplacement et orientation des bâtonnets sur les bois. Il apparaît donc que l'exécution des fermes a été réalisée par les

29. Outil dont la lame métallique est recourbée à son extrémité afin de graver les bois. 
mêmes équipes de charpentiers, avec probablement une équipe spécifique pour la réalisation des fermes principales, marquées distinctement. Il est vraisemblable aussi qu'une autre équipe était dévolue à la réalisation des éléments du dispositif longitudinal. En effet, les éléments longitudinaux ont leur marquage propre, distinct de celui des fermes et réalisé à l'aide d'un traceret ${ }^{30}$. Si les écharpes ne sont pas marquées, en revanche, dans chaque travée, les liernes inférieures sont marquées I et les liernes supérieures II, sur leur face inférieure, à l'ouest comme à l'est, avec un report sur le poinçon. Aucune marque n’a été relevée sur les sablières à part quelques signes d'appareillage ${ }^{31}$. Les pannes ont aussi leur numérotation propre avec des marques d'assemblage au droit de chaque enture. Chaque cours de pannes présente une suite numérique progressive de bâtonnets qui évolue de I à IIIIIIII (8), de l'est vers l'ouest. Ici, un système de contremarque existait : les marques du versant sud sont barrées d'un trait pour les distinguer de celles du versant nord.

Si ces différentes séries témoignent du travail de plusieurs équipes spécifiques, le plan de marquage ne laisse cependant entrevoir qu'une seule campagne de mise en œuvre, cohérente et bien ordonnée, même si cela ne contredit pas l'hypothèse d'un chantier étalé sur deux ans.

\subsection{LE LIGNAGE ET LE PIQUAGE}

Plusieurs traits de lignage ${ }^{32}$ subsistent en sous-face des chevrons. Réalisés à l'aide d'un cordeau imprégné de noir de charbon, tendu et frappé contre la face du bois, ces traits délimitent, selon un même axe, les mortaises destinées à l'entrait retroussé, au faux entrait et à l'enfourchement de tête. Quand le chevron n'est pas trop courbe, le lignage présente deux traits parallèles continus, encadrant les flancs de ces trois mortaises jusqu'en tête du chevron. Lorsque le chevron est trop courbe et que ces mortaises ne peuvent être alignées, le lignage présente un seul trait, passant par le flanc d'une mortaise puis par le flanc opposé de la suivante. Parfois ce trait unique sort du bois dans une courbure trop prononcée.

Ce lignage est donc basé sur l'alignement du flanc des mortaises, non de leur axe comme cela se pratique aussi au $\mathrm{XIII}^{\mathrm{e}}$ siècle $^{33}$. Il est destiné exclusivement aux mortaises et non aux entailles des mi-bois comme on le voit sur les fermes intermédiaires où le trait délimitant les mortaises du faux entrait ne passe pas par l'entaille du mi-bois de l'arbalétrier. Ce lignage existe aussi en sous-face des liernes longitudinales sous la forme de deux traits parallèles encadrant les tenons des extrémités, sans passer là aussi par les entailles à mi-bois des écharpes.

On observe aussi, au droit de nombreux assemblages, les tracés de piquage ${ }^{34}$ au traceret destinés à définir le profil des assemblages à tailler.

\footnotetext{
30. Traceret : petite pointe métallique.

31. Traits d'appareillage : tracés recouvrant un assemblage sur les deux pièces pour vérifier si leur jonction est correcte.

32. Le lignage consiste à tracer des lignes sur la face d'un bois, sur sa longueur, pour délimiter les flancs des assemblages à tailler selon un axe commun.

33. ÉPAud 2007, p. 58.

34. Piquage : opération réalisée après le lignage qui consiste à tracer, avec un fil à plomb et un traceret, par superposition des bois, l'emplacement des assemblages suivant leurs bords latéraux, perpendiculaires aux traits de lignage.
}

\subsection{LE LEVAGE}

Le levage de la charpente de la nef a très vraisemblablement bénéficié d'un plancher de travail sur toute la longueur du vaisseau, établi en haut des murs, à hauteur des sablières, et soutenu par un échafaudage depuis le sol. Les bois auraient été montés un à un à l'aide d'un engin de levage jusqu'à ce plancher sur lequel ils étaient assemblés et chevillés, suivant leur marquage, pour constituer une ferme qui était ensuite dressée à son emplacement définitif. Toutes les fermes auraient été ainsi levées une par une, depuis le pignon ouest jusqu'au pignon est, d'après la face de marquage et le sens d'enfoncement des chevilles. Après la pose des dernières fermes à l'est, les pannes ont été posées en continu de l'est vers l'ouest, d'après leurs entures biaises situées toujours 0,20 à $0,30 \mathrm{~m}$ à l'ouest d'une ferme principale, avec les poteaux latéraux et leur entretoise, insérés dans les fermes principales grâce à leurs mi-bois et destinés au soutien des pannes. Le chevillage des chevrons aux pannes et l'insertion du dispositif longitudinal axial ont ensuite été réalisés suivant deux orientations, depuis chaque pignon jusqu'à leur jonction à la 7e travée. En effet, d'une part, le chevillage des chevrons aux pannes a été pratiqué selon deux techniques distinctes, tant sur la nef que sur les bas-côtés des deux versants : perpendiculairement aux pannes sur les trois dernières travées ouest, et verticalement sur les autres. Il s'agit d'habitudes propres à deux équipes distinctes qui démontrent que deux chantiers se sont rejoints à la $7^{\text {e }}$ travée et que les bas-côtés ont été levés conjointement aux fermes de la nef. D’autre part, les deux liernes de la septième travée ont été insérées dans le poinçon de F8 dans des mortaises surcreusées en longueur pour leur insertion en bascule (fig. 19), témoignant d'un raccord en cet endroit dans le montage de ce dispositif axial, levé dans deux sens opposés, à partir de chaque pignon.

Divers artefacts témoignent des opérations de levage. De nombreux entraits retroussés ont été retaillés au cours du montage, en raison de leur courbure, pour garantir le passage des écharpes. Des cales ont aussi été insérées sous des chevrons qui ne reposaient pas directement sur les pannes. Plusieurs bois conservent, sur une de leurs arêtes et au premier tiers de leur longueur, une entaille profonde pour l'accroche des cordes du levage. On observe aussi, aux extrémités de nombreuses liernes et écharpes, des trous de 0,22 $\mathrm{m}$ de diamètre pour, probablement, la fixation de perches provisoires de maintien dans l'attente de leur assemblage au poinçon. D'autres trous sont présents, par paire, sur les flancs des blochets et des entraits retroussés, liés au dressage de la ferme. Enfin, tous les poinçons, les arbalétriers et les poteaux conservent des trous d'écheliers pour permettre aux charpentiers d'accéder aux parties hautes de la charpente au cours du levage.

Les chevilles en chêne, de section de $0,22 \times 0,22 \mathrm{~m}$ en tête et longues parfois de $0,45 \mathrm{~m}$, sont, en de multiples endroits, bloquées par un coin enfoncé dans leur queue, à l'opposé de leur enfoncement, pour garantir le blocage définitif de l'assemblage (fig. 20). 


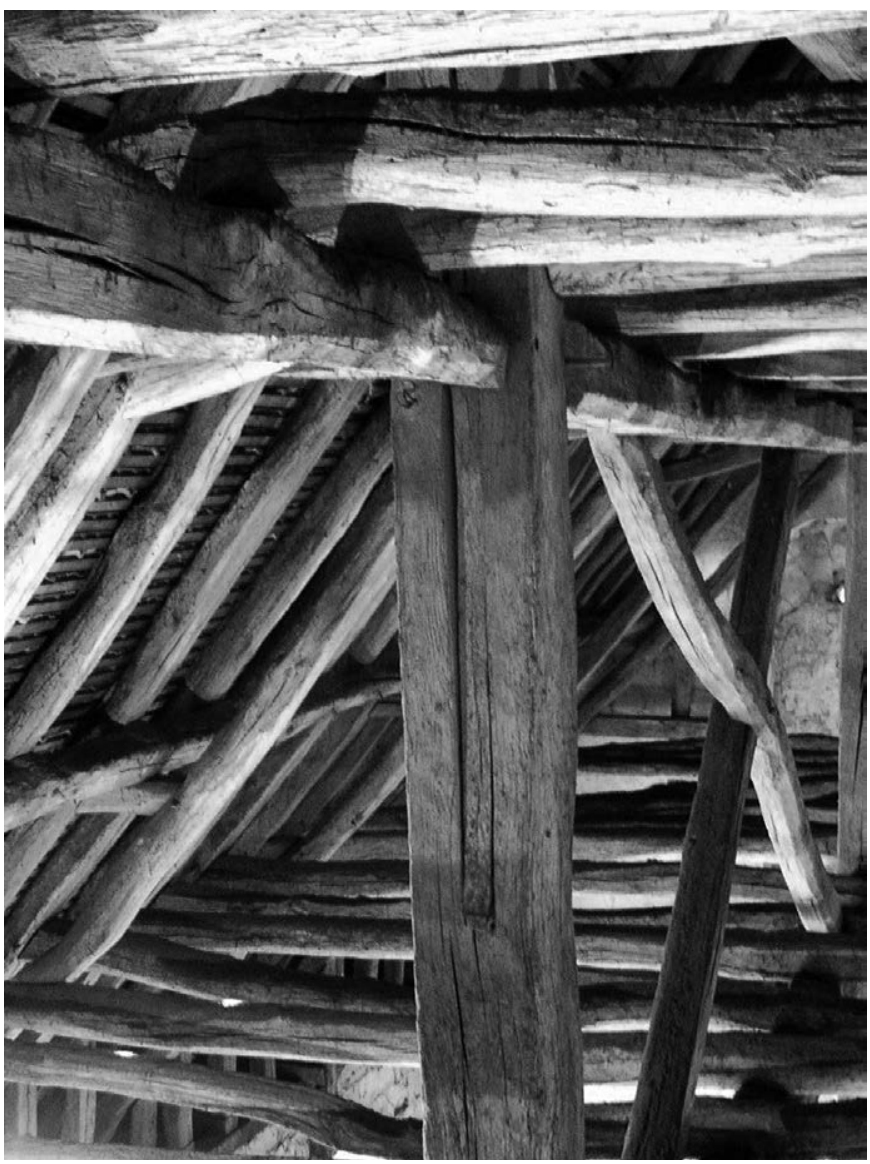

Fig. 19 Mortaise surcreusée en longueur pour l'insertion en bascule de la lierne axiale dans le poinçon de F8 (cl. F. E.).

\subsection{LA COUVERTURE}

Il est fortement probable que la couverture d'origine était en tuiles, comme cela a été constaté dans les sondages archéologiques situés à l'extérieur en pied des murs gouttereaux qui ont révélé la présence de nombreuses tuiles dans les niveaux de fondations de ces murs. En outre, dans la toiture rénovée en 2007, il a pu être observé de nombreuses tuiles de grand format, larges de $17 \mathrm{~cm}$, qui ont l'originalité de présenter un crochet décalé, à côté d'un trou destiné à un clou, pour l'éventuel réemploi de la tuile en cas de cassure du crochet.

\section{LA PLACE DE LA GRANGE SAINT-LAZARE DANS L'ÉVOLUTION DES CHARPENTES DU XIIII SIÈCLE}

L'implantation des abbayes cisterciennes dans l'Oise fut importante au XII ${ }^{\mathrm{e}}$ siècle avec la fondation de cinq abbayes d'hommes et quatre de femmes, filiales de Clairvaux et de Pontigny. Le développement de leur domaine foncier a donné naissance à de nombreux centres d'exploitation agricole, pourvus chacun d'une grange. On estime une création d'au moins 27 granges cisterciennes sur les plateaux de l'Oise aux XII ${ }^{\mathrm{e}}-\mathrm{XIII}^{\mathrm{e}}$ siècles $^{35}$

35. BONNET-LABORDERIE 1985.

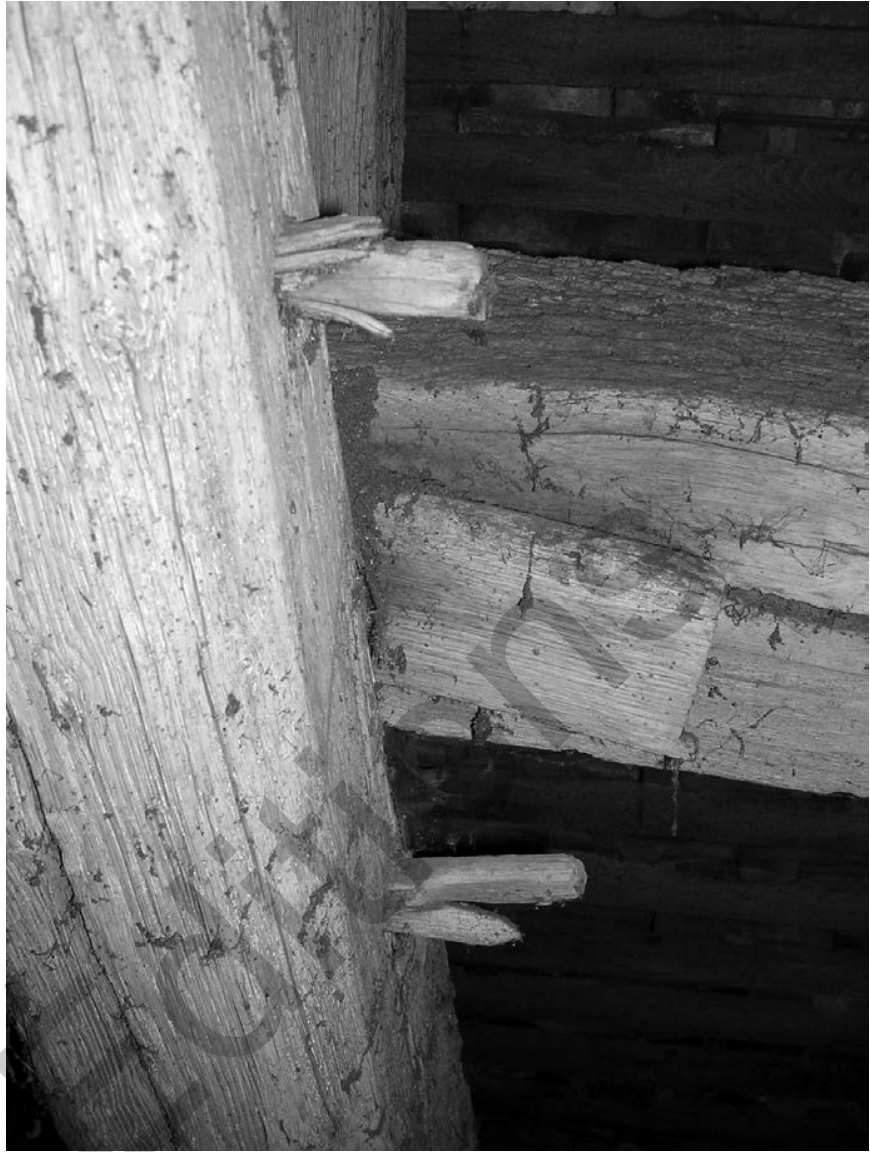

Fig. 20 Coins enfoncés dans la queue des chevilles et, en arrière-plan, cale pour l'ajustement d'une panne (cl. F. E.).

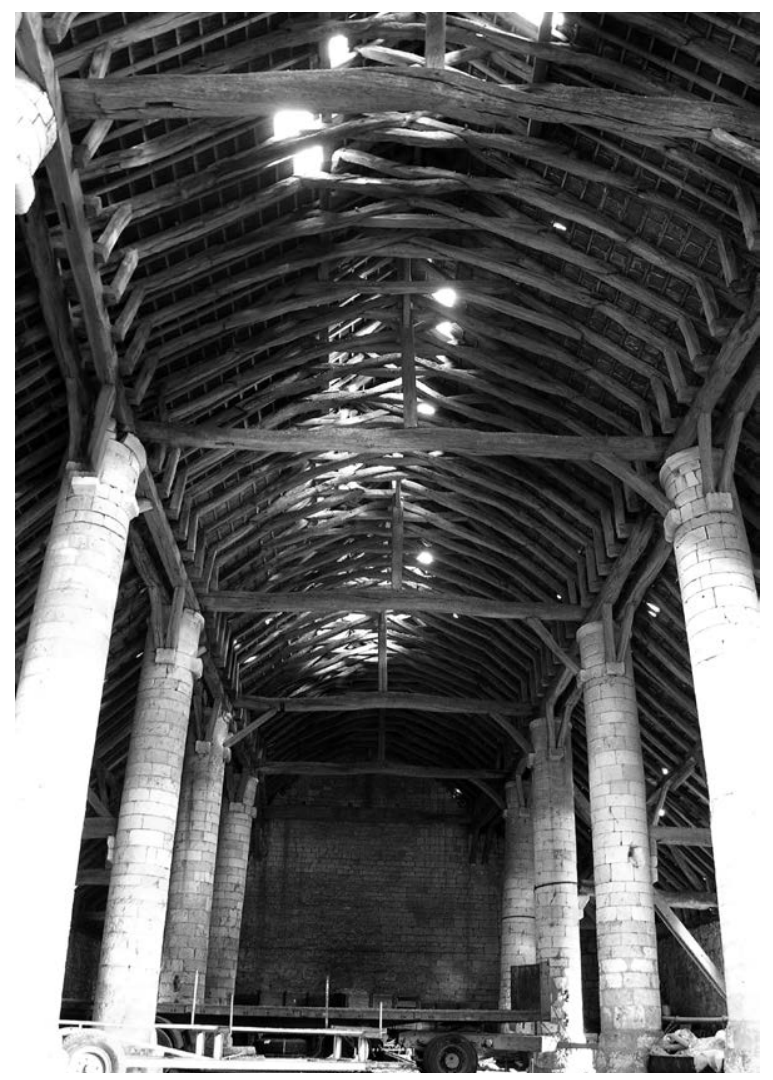

Fig. 21 Grange de Warnavillers (Oise), datée de 1189-1190d (cl. F. E.). 
Cathédrale de Paris, nef, vers 1220 (C.R.M.H. 1982, D6988)

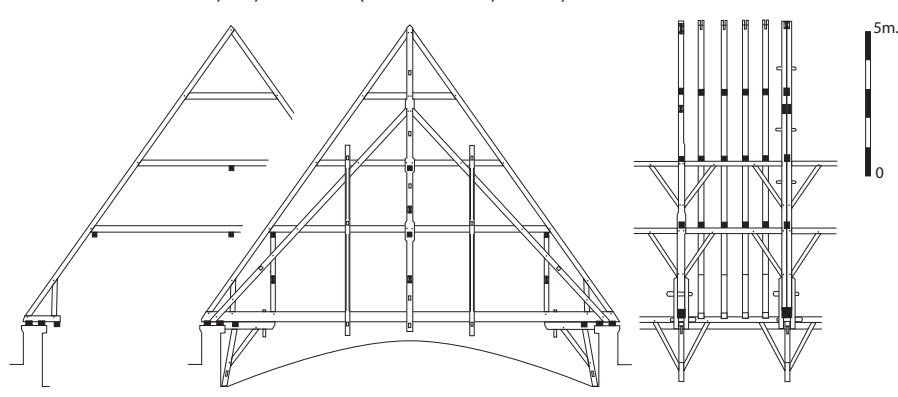

Cathédrale de Rouen, nef, 1227-1232d (Epaud 2007, p. 463)

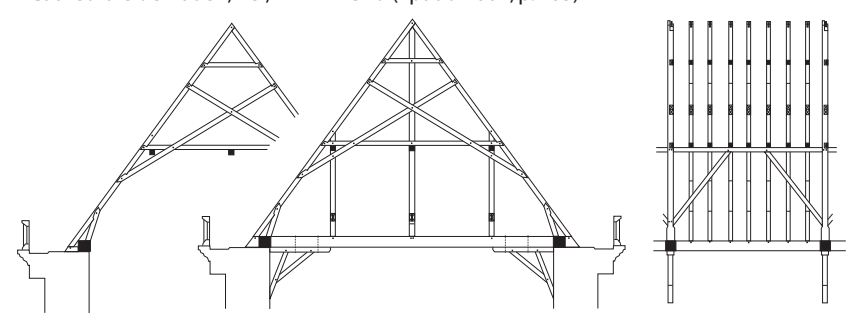

Cathédrale de Meaux, début du XIIle siècle (C.R.M.H. 1982, D6985)

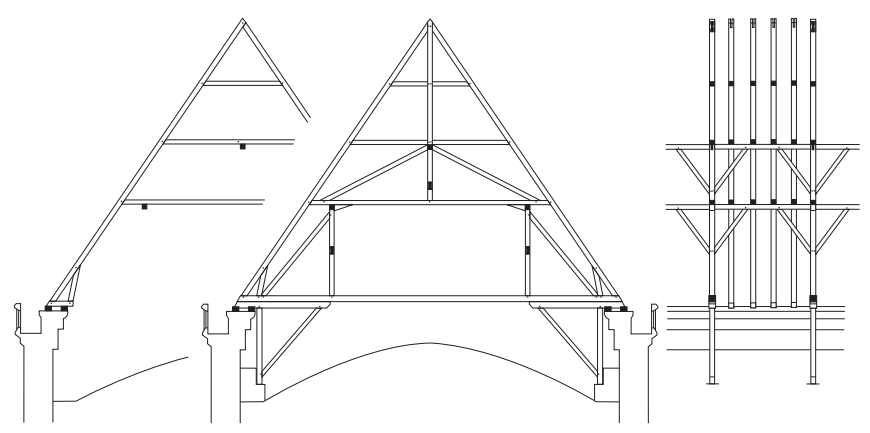

Cathédrale d'Auxerre, 1234-1236d (C.R.M.H. 1982, D6980-82)

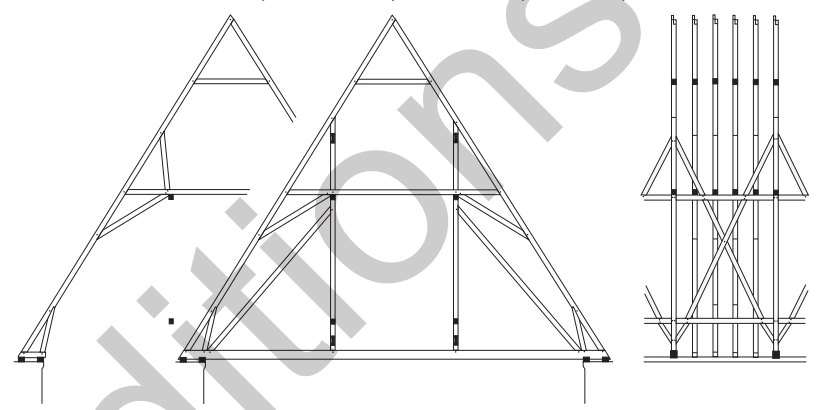

Paris, prieuré Saint-Martin-des-Champs, 1215-1220d (C.R.M.H. 1982, D6944-43)

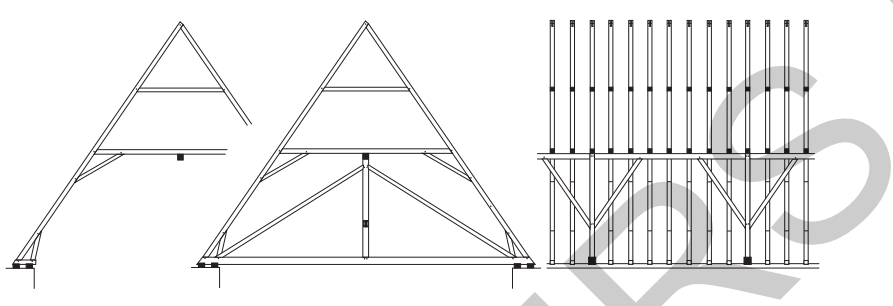

Collégiale de Mantes, début du XIIle siècle (C.R.M.H. 1982, D6909-10)

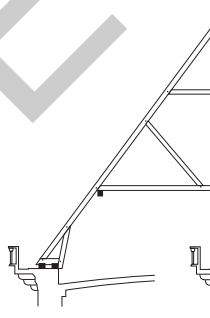

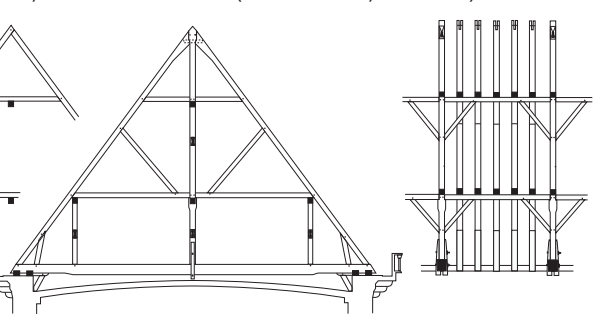

Fig. 22 Charpentes pourvues de dispositifs de soutien des fermes secondaires, début XIIIe siècle.

dont seules quelques-unes subsistent ${ }^{36}$, notamment la grange de Warnavillers, datée de 1189-1190d, la plus ancienne connue en France à l'heure actuelle ${ }^{37}$.

La grange de Saint-Lazare est comparable à ces granges cisterciennes $\mathrm{du} \mathrm{XIII}^{\mathrm{e}}$ siècle dans son plan et ses dimensions. Celles-ci ont un plan rectangulaire de $50 \times 18 \mathrm{~m}$ en moyenne, orientées nord-est, avec un vaisseau central bordé de deux bas-côtés, séparés par deux files de grandes arcades maçonnées retombant sur des piles quadrangulaires ${ }^{38}$, sauf pour Mauregard et Froidmont où les fermes reposent sur des poteaux en bois. Ces piles carrées semblent caractéristiques de l'Oise puisqu'elles ne se rencontrent que rarement dans les granges des autres régions. En Normandie comme en Île-de-France, les arcades retombent

36. Warnavillers, Fourcheret, Froidmont, Eraine, Fay.

37. Celle de la Haute-Croisille, dans l'Eure, pourrait être de la première moitié du XII ${ }^{e}$ siècle mais ne subsistent que les poteaux et les sablières.

38. La présence de grandes arcades sur piles quadrangulaires s'observe dans les granges de Troussures, Fourcheret, Fay, Stains et de Vaulerent, dépendantes de l'abbaye de Chaâlis ou celle de Grandmesnil, dépendante de Froidmont. sur des piles circulaires ${ }^{39}$, mais le plus souvent, la charpente repose sur des poteaux en bois. La toiture, dont les versants descendent très bas sur les gouttereaux, est comprise entre deux murs pignons, épaulés par des contreforts. La porte charretière s'ouvre presque toujours au mur pignon sud-ouest, dans l'axe de la nef ou d'un bas-côté, avec rarement une sortie dans l'autre pignon. Les entrées latérales dans les murs gouttereaux sont encore rares et ne se généralisent qu'à partir du XIV e siècle. Des baies étroites sont toujours présentes en haut des pignons pour la ventilation et la lumière. L'architecture de la grange de SaintLazare s'apparente donc à celle des granges cisterciennes et il ne semble donc pas exister de modèle de grange propre aux ordres hospitaliers ou aux ordres monastiques.

Concernant la structure de la charpente, la grange de Saint-Lazare s'inscrit dans le schéma évolutif des charpentes à chevrons-formant-fermes du premier tiers du XIII ${ }^{\mathrm{e}}$ siècle. Elle succède au type de charpentes tramées à chevrons-formant-fermes où les fermes sont indépendantes, sans dispositif

39. Abbaye d'Ardenne, prieuré de Perrières dans le Calvados, abbaye de Maubuisson dans le Val-d'Oise. 


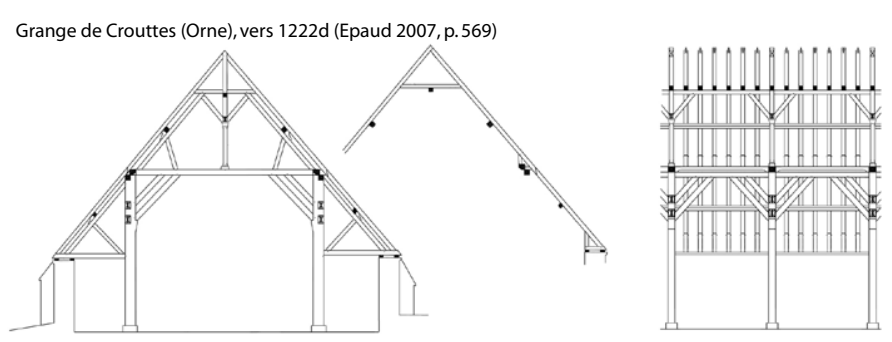

Halles de Saint-Pierre-sur-Dives (Calvados), première moitié du XIIle siècle (Epaud 2007, p. 575)

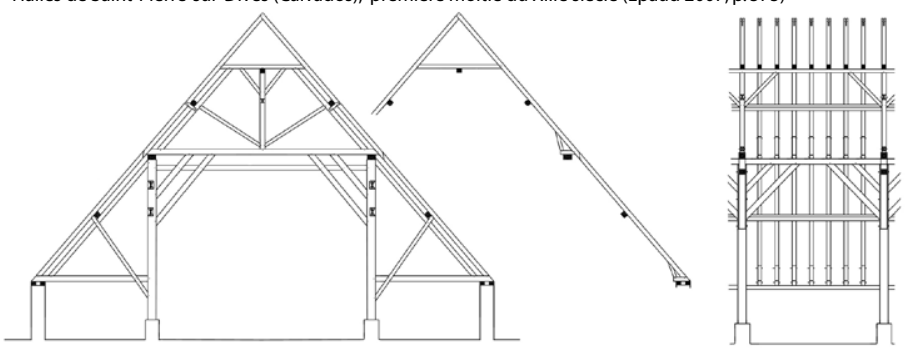

Grange du Val-de-la-Haye (Seine-Maritime), 1216-1220d (Epaud 2007, p. 553)

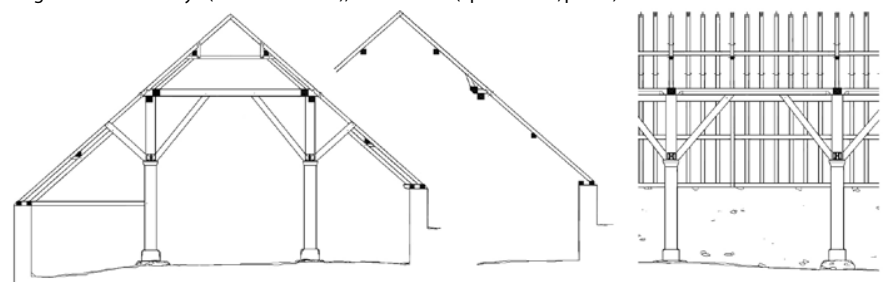

Grange de Heurteauville (Seine-Maritime), 1237-1243d (Epaud 2007, p. 565)

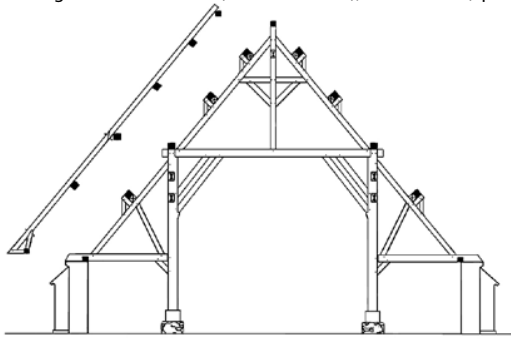

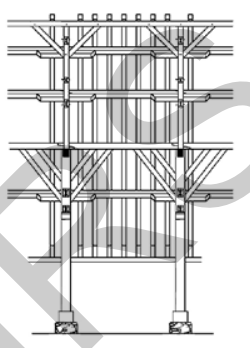

Grange de l'abbaye de Bonport, Pont-de-l'Arche (Eure), 1228d (Epaud 2007, p. 512)

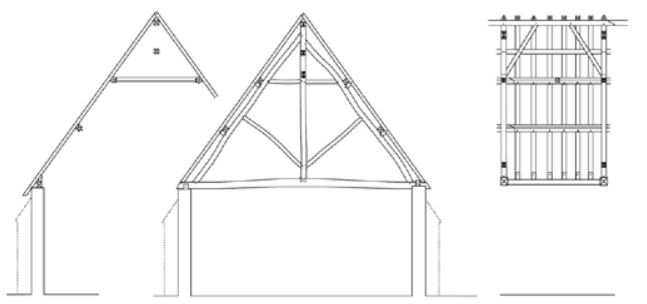

Grange de l'abbaye de Maubuisson, Saint-Ouen-l'Aumône (Val d'Oise), 1234-1264d (Dietrich et Gaultier 20001-2001, p. 117-118)

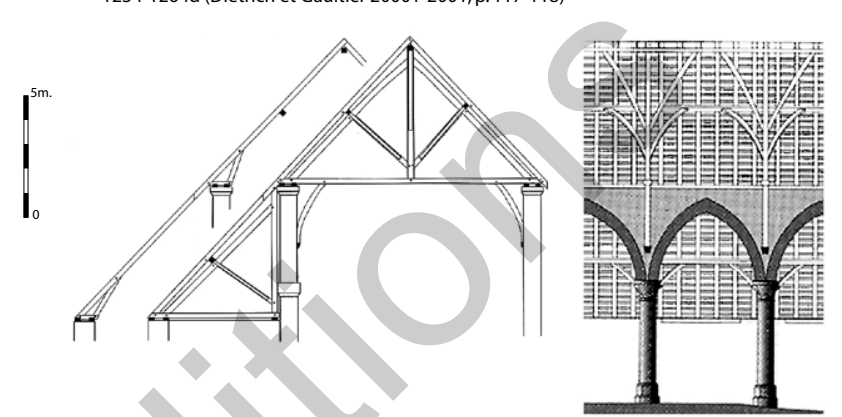

Grange du prieuré, Longpont-sur-Orge (Essonne) (๑ Inventaire général, ADAGP)

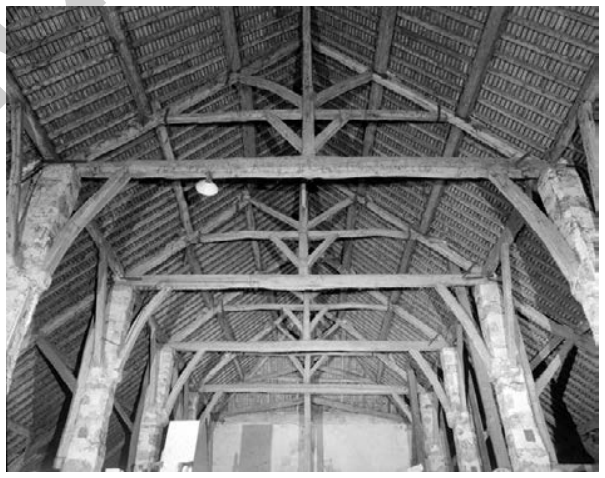

Fig. 23 Charpentes de granges du XIII' siècle.

longitudinal, avec une répartition homogène des charges le long des murs. L'unique représentant conservé de ce type antérieur est la grange de Warnavillers (Oise), datée de 1189-1190d (fig. 21) dont la structure est similaire en tout point à celle des charpentes des églises de la fin du XII ${ }^{\mathrm{e}}$ siècle et du début du XIII ${ }^{e}$ siècle. La grange de Froidmont (Bailleul-sur-Thérain, Oise) présente aussi dans sa charpente de nombreux réemplois qui témoignent d'une charpente primitive à chevrons-formantfermes, du XII ${ }^{\mathrm{e}}$ siècle, similaire à celle de Warnavillers.

Saint-Lazare se rattache à une famille de charpentes des années 1210-1230, spécifique des grands édifices gothiques du Bassin parisien (fig. 22). Leur structure répond aux contraintes de l'architecture gothique c'est-à-dire aux grandes portées et à la nécessité de réduire les poussées latérales sur les murs minces, percés de grandes verrières. Elle s'applique dans certains cas à concentrer les charges au droit des piles pour libérer les travées de ces poussées et permettre ainsi l'ouverture des murs de grandes fenêtres comme à la cathédrale de Rouen. Ce type de charpente présente donc des pièces longitudinales pour soutenir les fermes secondaires et transmettre les charges aux fermes principales, assises sur les points forts des maçonneries. Cette génération de charpentes est limitée aux années 1210-1230 et ne possède que peu de représentants. Elles se rencontrent dans la collégiale de Mantes, le chœur de l'église de Vétheuil dans le Val-d'Oise (1210-1212d), la nef et le chœur de la cathédrale de Paris (1220d), la nef de la cathédrale de Rouen (1227-1232d), le chœur de la cathédrale de Meaux (premier tiers XIII ${ }^{\mathrm{e}}$ siècle), la cathédrale d'Auxerre (1234-1236d), le grand dortoir de l'abbaye de Royaumont dans le Val-d'Oise (vers 1220). Il n'est pas étonnant de rencontrer un témoin de ce type dans une grange où les contraintes sont identiques à celles des grandes églises gothiques. En effet, du fait de la grande portée du vaisseau central et des files d'arcades, la charpente se doit de reporter ses charges au droit des piles, tout en limitant les poussées latérales dans les travées pour éviter que les arcades ne se déforment. Le maître d'œuvre de Saint-Lazare connaissait donc les dispositifs en vigueur à l'époque sur les cathédrales gothiques pour répondre à ces contraintes. Ces dispositifs ont pourtant été appliqués dans peu de granges. Dans l'état actuel des connaissances, outre Saint-Lazare, seules la grange 
de Crouttes (Orne) (1222d) et les halles de Saint-Pierre-surDives (Calvados) (disparue), appartiennent à cette génération de charpentes (fig. 23). Ces dernières présentent des pannes et une lierne axiale pour soutenir les fermes secondaires et reporter leurs charges sur les fermes principales, en appui sur des poteaux. Dans les autres granges du XIII ${ }^{\mathrm{e}}$ siècle, on observe majoritairement des charpentes à fermes et pannes qui se caractérisent par des fermes principales assises au droit des piliers ou des poteaux, et des chevrons simplement chevillés aux pannes. Il s'agit donc d'une structure simplifiée, dépourvue de ferme secondaire, et donc plus rapide à mettre en œuvre. Dans l'Oise et le Val-d'Oise, les granges abbatiales du XIII ${ }^{\mathrm{e}}$ siècle de Fourcheret, Troussures, Fay (Saintines), Vaumoise, Azémont (Crépy-en-Valois) $^{40}$, Saint-Ouen-l'Aumône ${ }^{41}$ et Tremblayen-France ont adopté ces structures à fermes et pannes, comme celle de Longpont-sur-Orge dans l'Essonne, détruite récemment. En Normandie, la charpente à fermes et pannes apparaît dans la grange du Val-de-la-Haye dès 1211-1220d à un stade expérimental où l'insertion des pannes dans les fermes principales n'est pas encore maîtrisée. On la retrouve à un stade plus évolué dans la grange de l'abbaye de Bonport en 1228d, celles de Heurteauville (1237-1243d) et de l'abbaye Notre-Dame d'Ardenne ${ }^{42}$ où les fermes principales réceptionnent les pannes sur de véritables arbalétriers. Leur diffusion dans les années
1220-1230 n'est pas limitée qu'aux granges et s'observe aussi dans les églises gothiques en Normandie ${ }^{43}$, dans l'Essonne ${ }^{44}$ et particulièrement dans le Val-d'Oise ${ }^{45}$. En effet, ce type de charpente répond parfaitement aux contraintes de l'architecture gothique par sa légèreté, son mode de répartition des charges, son faible coût et sa facilité de mise en œuvre.

Saint-Lazare utilise donc des principes structurels en usage essentiellement dans les cathédrales et les grandes églises gothiques de sa période, ce qui confirme le très haut niveau de compétence de son maître d'œuvre, confirmé par ailleurs par l'exceptionnelle complexité des assemblages. Il n'est pas interdit de penser, au regard des autres granges qui expérimentent aussi à la même époque l'insertion des pannes dans les chevronsformant-fermes, que ces granges ont pu servir de laboratoire, à la fin du XII $^{\mathrm{e}}$ et au début du XIII ${ }^{\mathrm{e}}$ siècle, pour la conception de charpentes mieux adaptées aux supports de piles successives et donc aux nouvelles exigences de l'architecture gothique. Les premières charpentes à fermes et pannes apparaissent en effet dans des granges, en Normandie, vers 1220, dans l'état actuel des connaissances, avec notamment plusieurs exemples de charpentes mixtes, associant le concept des chevrons-formant-fermes et des fermes et pannes, avant que ces dernières connaissent une très large diffusion, particulièrement dans l'architecture religieuse francilienne, vers 1220-1230.

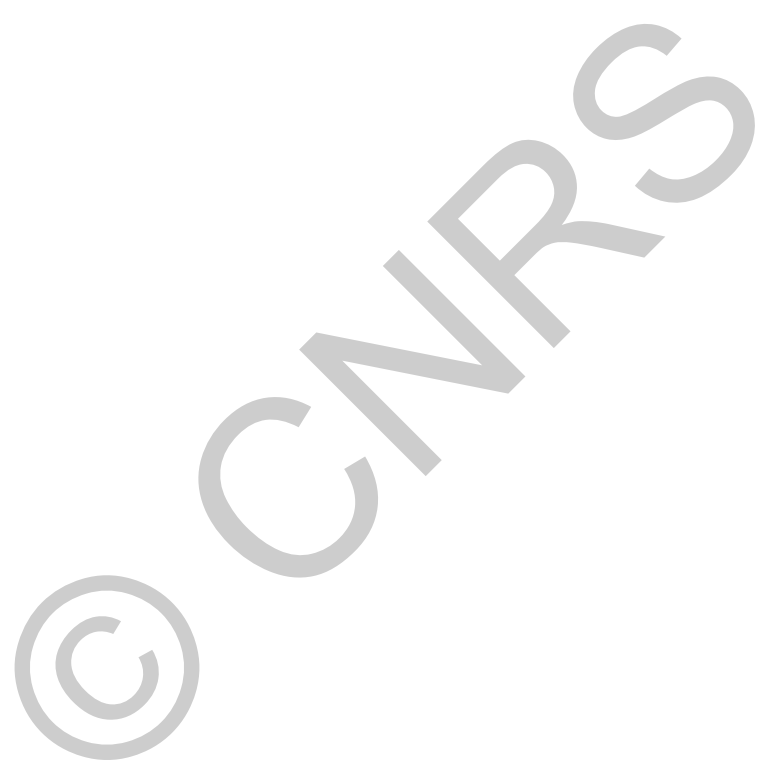

40. FranÇOIS 1980.

41. Dietrich et Gaultier 2000-2001.

42. ÉPAud 2007, p. 193.

43. Abbatiale de Fécamp (76) vers 1217-1223d; réfectoire de Bonport à Pont-de-l'Arche (27), 1240-1241d.

44. Vert-le-Petit dans l'Essonne, 1221-1222d.

45. Champagne-sur-Oise, 1234d; Fontenay-en-Parisis, vers 1250; Marlyla-Ville, 1262-1265d; Bessancourt, second quart XIII' siècle; Courcelles-surViosne, second quart XIII' siècle; Montreuil-sur-Epte, second quart XIII' siècle (Épaud et Bernard 2008). 


\section{BIBLIOGRAPHIE}

BHAHa J., JanÀK K. et RUZicka P.

2008, «Les traces du travail du bois» dans Hoffsummer P. et Eeckhout J. (dir.), Matériaux de l'architecture et toits de l'Europe, Les dossiers de l'IPW, t. 6, p. 119-139.

BLARY F.

1989, Le domaine de Chaalis, XII ${ }^{e}-\mathrm{XIV} V^{e}$ siècles, Paris, éditions du CTHS.

BONNET-LABORDERIE P.

1985, «La grange cistercienne, une architecture rurale médiévale», dans Blé et Patrimoine, Bulletin du Groupe d'Étude des Monuments et Euvres d'art du Beauvaisis, no 24, p. 9-20.

2002, «Un ensemble hospitalier du Moyen Âge. La maladrerie Saint-Lazare de Beauvais", Bulletin du Gemob, 107, Beauvais.

Cayez M.-J.

2007 «La maladrerie Saint-Lazare de Beauvais. Approche archéologique d'un établissement hospitalier médiéval", dans Étude des lépreux et des léproseries au Moyen Âge dans le nord de la France, B. Tabuteau (dir.), Amiens, Publication du Cahmer, vol. 20, p.151-184.

CRMH

1982, Charpentes, XIII siècle, vol. 2, Paris, Ministère de la Culture, Direction du Patrimoine.

Dietrich A. et Gaultier M., 2000-2001, «La charpente de la grange abbatiale de Maubuisson (Saint-Ouenl'Aumône, Val d'Oise)", Archéologie médiévale, t. 30-31, p. 109-132.
Dimier A.,

1975, «Les belles granges cisterciennes du département de l'Oise", Archéologia, $\mathrm{n}^{\circ} 85$, août 1975 , p. 49-61.

Durvin, P.

1975, La maladrerie Saint-Lazare de Beauvais, Bulletin de la Société nationale des antiquaires de France, Beauvais.

EDELMAN J.

1979, Une grande léproserie médiévale: Saint-Lazare de Beauvais, du XII ${ }^{e}$ au $\mathrm{XV}$ siècle, mémoire de maîtrise d'histoire (dir. J. Heers), dactylographiée, Université Paris X Nanterre.

ÉPAud F.

2007, De la charpente romane à la charpente gothique en Normandie. Évolution des structures et des techniques de charpenterie aux XII ${ }^{e}$-XIII ${ }^{e}$ siècles, Caen, Publications $\mathrm{du}$ Crahm.

ÉPAUd F. et BERNARD V.

2008, "L'évolution des charpentes d'églises du Val-d'Oise, du XI ${ }^{\mathrm{e}}$ au xx ${ }^{\mathrm{e}}$ siècle», Revue archéologique du Centre de la France, t. 47, mis en ligne le 18 mai 2009. URL : http:// racf.revues.org/index1205.html.

\section{FÉmolant J.-M.}

2007, «Saint-Lazare de Beauvais : l'apport des résultats du diagnostic archéologique préventif sur la ferme de la maladrerie» dans Étude des lépreux et des léproseries au Moyen Âge dans le nord de la France, B. Tabuteau (dir.), Amiens, Publication du Cahmer, vol. 20, p. 27-28.

2004, «Un diagnostic archéologique sur la maladrerie Saint-Lazare», dans Hôpitaux et maladreries au Moyen Âge : espace et environnement, actes du colloque international d'Amiens-Beauvais (22, 23, 24 novembre 2002), Amiens, Publication du Cahmer, vol. 17, p. 353-361.

2003, «Beauvais : maladrerie Saint-Lazare», dans Bilan scientifique de la région Picardie, Amiens, Service régional de l'Archéologie, Drac Picardie, p. 66-67.

FÉmolant J.-M. et Veyssier D.

2006, «Beauvais : maladrerie Saint-Lazare», dans Bilan scientifique de la région Picardie, Amiens, Service régional de l'Archéologie, Drac Picardie, p. 66.

FrançOIS J.-L.

1980, «Trois granges médiévales méconnues dans le canton de Crépy-en-Valois (Oise)", Revue archéologique de l'Oise, vol. 20, p. 17-20.

http://www.persee.fr/web/revues/home/ prescript/article/pica_0752-5648_1980_ num_20_1_1136

LEBLOND V.

1922, Cartulaire de la Maladrerie SaintLazare de Beauvais, Publication de la société académique de l'Oise, Paris, éditions Champion.

1926, Les lépreux de Beauvais. La Maladrerie de Saint-Lazare au XVI ${ }^{e}$ siècle d'après les comptes d'administrations, Beauvais, Imprimerie départementale de l'Oise.

Le Digol Y. et Bernard V.

2006, Grange de la léproserie Saint-Lazare, Beauvais (60), Rapport d'étude dendrochronologique, Rennes.

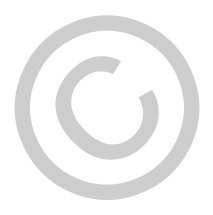

